\title{
Finite element approximation of a phase field model for tumour growth
}

\author{
Joe Eyles*, Robert Nürnberg ${ }^{\dagger}$ and Vanessa Styles*
}

\begin{abstract}
We consider a fully practical finite element approximation of a diffuse interface model for tumour growth that takes the form of a degenerate parabolic system. In addition to showing stability bounds for the approximation, we prove convergence, and hence existence of a solution to this system in two space dimensions. Several numerical experiments demonstrate the practicality and robustness of the proposed method.
\end{abstract}

\section{Introduction}

Mathematical modelling is an important tool in the study and treatment of tumours, and as such is an area of research that continues to attract a great deal of interest, see for example [3, 4, 7] and the references therein. The mathematical model we consider is one in which the tumour is modelled as a continuum using partial differential equations (PDEs), rather than an individual-based cell model in which a collection of discrete cells undergo stochastic or deterministic behaviour. There is a wealth of literature on continuum tumour growth models, with some of the earliest dating from the 1970s, see [17, 18] in which the models take the form of free boundary problems, with the free boundary representing the boundary between the interior and exterior of the tumour. Free boundary problems for tumour growth have been successfully approximated using a number of diffuse interface approaches, see for instance [6, 8, 14, 16, 15]. In this paper, we analyse the two dimensional version of the diffuse interface model for tumour growth presented in [12]. It approximates the following free boundary problem, which is one of the simplest

\footnotetext{
*Department of Mathematics, University of Sussex, Brighton BN1 9HQ, UK

${ }^{\dagger}$ Department of Mathematics, University of Trento, Trento, Italy
} 
mathematical descriptions of the growth and death of a tumour:

$$
\begin{array}{rr}
\Delta u=1, & \text { in } \widetilde{\Omega}(t), \\
\nabla u \cdot n+\frac{u}{\alpha}=Q, & \text { on } \Gamma(t), \\
V=\beta \kappa+\frac{u}{\alpha}, & \text { on } \Gamma(t) .
\end{array}
$$

Here $\Gamma(t)=\partial \widetilde{\Omega}(t)$ is an evolving curve in $\mathbb{R}^{2}$, with its interior $\widetilde{\Omega}(t)$ representing the tumour cells, and $u$ corresponds to the tissue pressure in the tumour. Moreover, $V$ is the velocity of $\Gamma(t)$ in the direction of the normal $n$, while $\kappa$ denotes the curvature of $\Gamma(t)$. The constant $Q \in \mathbb{R}_{>0}$ represents a surface source, while $\alpha, \beta \in \mathbb{R}_{>0}$ are regularisation parameters. The free boundary problem (1.1) was formally derived in [12] as a thin-rim limit of a reaction-diffusion system involving living and dead tumour cells, a nutrient and a Darcy's law for the velocity and pressure. The advantage of the limiting problem (1.1) is that the number of unknowns is reduced to just the free boundary and the pressure. Mathematically, (1.1) describes the evolution of the closed curve $\Gamma(t)$ via forced curvature flow, where the forcing depends on the solution of an elliptic PDE in its interior.

A possible diffuse interface approximation of $(1.1)$ is given as follows. Let $\Omega$ be a fixed, bounded domain in $\mathbb{R}^{2}$, let $\vartheta \in\{0,1\}$ and let $\varepsilon \in \mathbb{R}_{>0}$. Then find a phase field $\varphi_{\varepsilon}: \Omega \rightarrow \mathbb{R}$, and a pressure $u_{\varepsilon}: \Omega \rightarrow \mathbb{R}$ such that

$$
\begin{aligned}
\varepsilon^{2} \vartheta \partial_{t} u_{\varepsilon}-\nabla \cdot\left(\zeta\left(\varphi_{\varepsilon}\right) \nabla u_{\varepsilon}\right)+\frac{1}{\varepsilon} \delta\left(\varphi_{\varepsilon}\right)\left(\frac{u_{\varepsilon}}{\alpha}-Q\right)+\zeta\left(\varphi_{\varepsilon}\right)=0 & \text { in } \Omega, \\
\varepsilon \partial_{t} \varphi_{\varepsilon}-\varepsilon \beta \Delta \varphi_{\varepsilon}+\frac{\beta}{\varepsilon} \partial W\left(\varphi_{\varepsilon}\right)-\frac{c_{W}}{\alpha} u_{\varepsilon} \ni 0 & \text { in } \Omega, \\
\zeta\left(\varphi_{\varepsilon}\right) \nabla u_{\varepsilon} \cdot \nu=\nabla \varphi_{\varepsilon} \cdot \nu=0 & \text { on } \partial \Omega,
\end{aligned}
$$

where $\nu$ denotes the outer normal on $\partial \Omega$. In the above

$$
\zeta(s)=\frac{1+s}{2} \quad \text { and } \quad \delta(s)=\frac{2}{\pi}\left(1-s^{2}\right) \quad s \in \mathbb{R},
$$

and $W$ denotes the double-obstacle potential

$$
W(s)=\frac{1}{2}\left(1-s^{2}\right)+I_{[-1,1]}(s) \quad s \in \mathbb{R},
$$

with $I_{[-1,1]}$ denoting the indicator function

$$
I_{[-1,1]}(s):= \begin{cases}+\infty & \text { for }|s|>1 \\ 0 & \text { for }|s| \leq 1\end{cases}
$$

see [5]. Moreover, $c_{W}=\frac{1}{2} \int_{-1}^{1} \sqrt{2 W(s)} \mathrm{d} s=\frac{\pi}{4}$ and $\partial W$ denotes the subdifferential of $W$, so that $\partial W(s)=-s+\partial I_{[-1,1]}(s)$.

The model $(1.2)$, with $\vartheta=0$, has recently been considered in [12]. There it was shown, using formal asymptotic analysis, that in the sharp interface limit, $\varepsilon \rightarrow 0$, the original 
problem (1.1) is recovered. Moreover, a finite element approximation of $(1.2)$, with $\vartheta=0$, was introduced, and several numerical simulations were presented. In this paper, we are interested in the numerical analysis of a suitable approximation of $(1.2)$. To this end, we first of all note that it is a simple matter, on using the results in [9], to extend the formal asymptotic analysis in 12 to the case $\vartheta=1$. In particular, (1.1) is recovered as the sharp interface limit of (1.2) with $\vartheta=1$, as $\varepsilon \rightarrow 0$. Moreover, what makes the analysis of 1.2 challenging is the presence of the degenerate coefficients $\delta\left(\varphi_{\varepsilon}\right)$ and $\zeta\left(\varphi_{\varepsilon}\right)$ in $(1.2 \mathrm{a})$. In the elliptic case, when $\vartheta=0$, it does not seem to be easily possible to prove convergence for the finite element approximation introduced in [12]. Hence, in this paper, we consider the case $\vartheta=1$, which changes $1.2 \mathrm{a}$ to a parabolic problem. Although there is no bio-physical basis for the added term $\varepsilon^{2} \partial_{t} u_{\varepsilon}$, it yields additional smoothness for $u_{\varepsilon}$ that enables us to derive a convergence proof. However, we note that the model of interest to bio-physicists is (1.1), which is not impacted by the additional term in the phase field model. We remark that (1.2) with $\vartheta=1$ is closely related to the problem studied in [10]. That is why many of the ingredients for our convergence proof are based on extending the techniques in [10] to the more complicated problem 1.2 .

The remainder of the paper is organised as follows. In Section 2 we present a weak formulation of $(1.2)$ and introduce a finite element approximation. We prove the wellposedness and a maximum principle for the discrete system. Convergence of the discrete solutions to a weak solution of $(1.2)$ is shown in Section 3 , and some numerical simulations are presented in Section 4 .

We end this section with a few comments about notation. The $L^{2}$-inner product on $\Omega$ is denoted by $(\cdot, \cdot)$. We adopt the standard notation for Sobolev spaces, denoting the norm of $W^{\ell, p}(\Omega)(\ell \in \mathbb{N}, p \in[1, \infty])$ by $\|\cdot\|_{\ell, p}$ and the semi-norm by $|\cdot|_{\ell, p}$. For $p=2$, $W^{\ell, 2}(\Omega)$ will be denoted by $H^{\ell}(\Omega)$ with the associated norm and semi-norm written, as respectively, $\|\cdot\|_{\ell}$ and $|\cdot|_{\ell}$. In addition, we adopt the standard notation $W^{\ell, p}(a, b ; X)$

$(\ell \in \mathbb{N}, p \in[1, \infty],(a, b)$ an interval in $\mathbb{R}, X$ a Banach space) for time dependent spaces with norm $\|\cdot\|_{W^{\ell, p}(a, b ; X)}$. Once again, we write $H^{\ell}(a, b ; X)$ if $p=2$. Furthermore, $C$ denotes a generic constant independent of the mesh parameter $h$ and the time step $\Delta t$, see below.

\section{Weak formulation and finite-element approxima- tion}

In what follows, we let $\vartheta=1$, fix a value $\varepsilon \in \mathbb{R}_{>0}$ and drop the subscript $\varepsilon_{\varepsilon}$ in $(1.2$ for notational convenience. We also fix a final positive time $T$.

The obstacle nature of 1.4 means that $1.2 \mathrm{~b}$ will lead to a variational inequality. To this end, we introduce the convex subset

$$
K=\left\{\eta \in H^{1}(\Omega)|| \eta \mid \leq 1 \text { in } \Omega\right\}
$$

of $H^{1}(\Omega)$. Then a weak formulation of 1.2 is given as follows. 
$\mathbb{P}$ Let $u(0)=u_{0} \in H^{1}(\Omega)$ and $\varphi(0)=\varphi_{0} \in K$. Then, for $t \in(0, T]$ find $(u(t), \varphi(t)) \in$ $H^{1}(\Omega) \times K$ such that

$$
\begin{aligned}
& \varepsilon^{2}\left(\partial_{t} u, \eta\right)+(\zeta(\varphi) \nabla u, \nabla \eta)+\frac{1}{\varepsilon \alpha}(\delta(\varphi) u, \eta)=\frac{Q}{\varepsilon}(\delta(\varphi), \eta)-(\zeta(\varphi), \eta) \quad \forall \eta \in H^{1}(\Omega), \\
& \varepsilon\left(\partial_{t} \varphi, \rho-\varphi\right)+\varepsilon \beta(\nabla \varphi, \nabla(\rho-\varphi))-\frac{\beta}{\varepsilon}(\varphi, \rho-\varphi) \geq \frac{c_{W}}{\alpha}(u, \rho-\varphi) \quad \forall \rho \in K .
\end{aligned}
$$

We consider the finite element approximation of $\mathbb{P}$ under the following assumptions on the mesh:

(A) Let $\Omega$ be a polygonal domain. Let $\left\{\mathcal{T}_{h}\right\}_{h>0}$ be a quasi-uniform family of partitionings of $\Omega$ into disjoint open simplices $\sigma$ with $h_{\sigma}:=\operatorname{diam}(\sigma)$ and $h:=\max _{\sigma \in \mathcal{T}_{h}} h_{\sigma}$, so that $\bar{\Omega}=\cup_{\sigma \in \mathcal{T}_{h}} \bar{\sigma}$. In addition, it is assumed that all simplices $\sigma \in \mathcal{T}_{h}$ are acute.

Associated with $\mathcal{T}_{h}$ is the finite element space

$$
S_{h}:=\left\{\eta_{h} \in C^{0}(\bar{\Omega})\left|\eta_{h}\right|_{\sigma} \text { is linear } \forall \sigma \in \mathcal{T}_{h}\right\} .
$$

We also introduce

$$
K_{h}:=S_{h} \cap K .
$$

Let $J$ be the set of nodes of $\mathcal{T}_{h}$ and $\left\{p_{j}\right\}_{j \in J}$ the coordinates of these nodes. Let $\left\{\chi_{j}\right\}_{j \in J}$, be the standard basis functions for $S_{h}$; that is $\chi_{j} \in S_{h}$ and $\chi_{j}\left(p_{i}\right)=\delta_{i j}$ for all $i, j \in J$. We denote by $I_{h}: C^{0}(\bar{\Omega}) \rightarrow S_{h}$ the Lagrange interpolation operator onto $S_{h}$. We denote the discrete $L^{2}$-inner product on $C^{0}(\bar{\Omega})$ by

$$
(u, v)_{h}:=\left(I_{h}(u v), 1\right) \quad u, v \in C^{0}(\bar{\Omega}),
$$

and we set $|v|_{h}^{2}:=(v, v)_{h}$.

In addition to $\mathcal{T}_{h}$, let $0=t_{0}<t_{1}<\ldots<t_{N-1}<t_{N}=T$ be a partitioning of $[0, T]$ into uniform time steps $t_{n}-t_{n-1}=\Delta t=T / N, n=1, \ldots, N$. Then we consider the following finite element approximation of $\mathbb{P}$.

$\mathbb{P}_{h}$ Let $u_{h}^{0}:=I_{h} u_{0}$ and $\varphi_{h}^{0}:=I_{h} \varphi_{0}$. Then, for $n=0, \ldots, N-1$, find $u_{h}^{n+1} \in S_{h}$ and $\varphi_{h}^{n+1} \in K_{h}$ such that

$$
\begin{aligned}
\frac{\varepsilon^{2}}{\Delta t}\left(u_{h}^{n+1}-\right. & \left.u_{h}^{n}, \eta_{h}\right)_{h}+\left(\zeta\left(\varphi_{h}^{n}\right) \nabla u_{h}^{n+1}, \nabla \eta_{h}\right)+\frac{1}{\varepsilon \alpha}\left(\delta\left(\varphi_{h}^{n}\right) u_{h}^{n+1}, \eta_{h}\right)_{h} \\
= & \frac{Q}{\varepsilon}\left(\delta\left(\varphi_{h}^{n}\right), \eta_{h}\right)_{h}-\left(\zeta\left(\varphi_{h}^{n}\right), \eta_{h}\right)_{h} \forall \eta_{h} \in S_{h} \\
\frac{\varepsilon}{\Delta t}\left(\varphi_{h}^{n+1}-\right. & \left.\varphi_{h}^{n}, \rho_{h}-\varphi_{h}^{n+1}\right)_{h}+\varepsilon \beta\left(\nabla \varphi_{h}^{n+1}, \nabla \rho_{h}-\nabla \varphi_{h}^{n+1}\right) \\
& -\frac{\beta}{\varepsilon}\left(\varphi_{h}^{n+1}, \rho_{h}-\varphi_{h}^{n+1}\right)_{h} \geq \frac{c_{W}}{\alpha}\left(u_{h}^{n+1}, \rho_{h}-\varphi_{h}^{n+1}\right)_{h} \quad \forall \rho_{h} \in K_{h} .
\end{aligned}
$$


We note that the discretisation in $(2.2)$ is chosen such that the linear system (2.2a) decouples from the variational inequality $2.2 \mathrm{~b}$. Hence in practice we can first solve (2.2a) to obtain $u_{h}^{n+1}$, and then find $\varphi_{h}^{n+1} \in K_{h}$ solving (2.2b).

In what follows, we will make use of the standard inequality

$$
\left|v_{h}\right|_{0}^{2} \leq\left|v_{h}\right|_{h}^{2} \leq 4\left|v_{h}\right|_{0}^{2} \quad v_{h} \in S_{h}
$$

Lemma 2.1. Let $u_{h}^{n} \in S_{h}$ and $\varphi_{h}^{n} \in K_{h}$. Then for $\Delta t<\frac{\varepsilon^{2}}{\beta}$ there exists a unique solution $\left(u_{h}^{n+1}, \varphi_{h}^{n+1}\right) \in S_{h} \times K_{h}$ to 2.2 .

Proof. The existence of a unique solution $u_{h}^{n+1}$ to $2.2 \mathrm{a}$ follows immediately from the fact that the system is linear, symmetric and positive definite. In addition, there exists a $\varphi_{h}^{n+1} \in K_{h}$ solving $2.2 \mathrm{~b}$ since this is the Euler-Lagrange variational inequality of the minimisation problem:

$$
\min _{z_{h} \in K_{h}}\left\{\frac{\varepsilon \beta}{2}\left|\nabla z_{h}\right|_{0}^{2}+\frac{\varepsilon}{2 \Delta t}\left|I_{h}\left(z_{h}-\varphi_{h}^{n}\right)\right|_{0}^{2}+\frac{\beta}{2 \varepsilon}\left(1-z_{h}^{2}, 1\right)_{h}-\frac{c_{W}}{\alpha}\left(u_{h}^{n+1}, z_{h}\right)_{h}\right\},
$$

for which the existence of a minimiser can be shown by a standard minimisation argument. For uniqueness we assume there exist two solutions $\varphi_{h, 1}^{n+1}$ and $\varphi_{h, 2}^{n+1}$ and set $\theta=\varphi_{h, 1}^{n+1}-\varphi_{h, 2}^{n+1}$. Taking $\eta_{h}=\varphi_{h, 2}^{n+1}$ in the variational inequality $(2.2 \mathrm{~b})$ for $\varphi_{h, 1}^{n+1}$ and vice versa, then subtracting one of the resulting inequalities from the other we obtain

$$
\frac{\varepsilon^{2}}{\Delta t}|\theta|_{h}^{2}+\varepsilon^{2} \beta|\nabla \theta|_{0}^{2}-\beta|\theta|_{h}^{2} \leq 0 \Rightarrow\left(\frac{\varepsilon^{2}}{\Delta t}-\beta\right)|\theta|_{h}^{2}+\varepsilon^{2} \beta|\nabla \theta|_{0}^{2} \leq 0,
$$

which yields uniqueness for $\Delta t<\frac{\varepsilon^{2}}{\beta}$.

\section{Convergence of the finite element scheme}

In this section, which makes use of many of the techniques in [10], we prove that as $h \rightarrow 0$ the solution of the finite element scheme $\mathbb{P}_{h}$ converges to the solution of $\mathbb{P}$. Here we fix $\varepsilon$, and assume that $\Delta t \leq C h^{2}$. All limits are taken as $h \rightarrow 0$ (and thus $\Delta t \rightarrow 0$ ). We introduce the notation

$$
\begin{aligned}
\varphi_{h}(t) & :=\frac{t-t_{n}}{\Delta t} \varphi_{h}^{n+1}+\frac{t_{n+1}-t}{\Delta t} \varphi_{h}^{n}, \quad t \in\left(t_{n}, t_{n+1}\right], \\
\varphi_{h}^{+}(t) & :=\varphi_{h}^{n+1}, \quad \varphi_{h}^{-}(t):=\varphi_{h}^{n}, \quad t \in\left(t_{n}, t_{n+1}\right],
\end{aligned}
$$

and similarly for $u_{h}(t), u_{h}^{+}(t)$ and $u_{h}^{-}(t)$. Furthermore we use ${ }^{(\star)}$ to denote an expression with or without the superscript $\star$. 
Rewriting (2.2) using the above notation gives, for $n=0, \ldots, N-1$ and for $t \in\left(t_{n}, t_{n+1}\right]$,

$$
\begin{aligned}
& \varepsilon^{2}\left(\partial_{t} u_{h}, \eta_{h}\right)_{h}+\left(\zeta\left(\varphi_{h}^{-}\right) \nabla u_{h}^{+}, \nabla \eta_{h}\right)+\frac{1}{\varepsilon \alpha}\left(\delta\left(\varphi_{h}^{-}\right) u_{h}^{+}, \eta_{h}\right)_{h}=\frac{Q}{\varepsilon}\left(\delta\left(\varphi_{h}^{-}\right), \eta_{h}\right)_{h}-\left(\zeta\left(\varphi_{h}^{-}\right), \eta_{h}\right)_{h}, \\
& \varepsilon\left(\partial_{t} \varphi_{h}, \rho_{h}-\varphi_{h}^{+}\right)_{h}+\varepsilon \beta\left(\nabla \varphi_{h}^{+}, \nabla \rho_{h}-\nabla \varphi_{h}^{+}\right)-\frac{\beta}{\varepsilon}\left(\varphi_{h}^{+}, \rho_{h}-\varphi_{h}^{+}\right)_{h} \geq \frac{c_{W}}{\alpha}\left(u_{h}^{+}, \rho_{h}-\varphi_{h}^{+}\right)_{h},
\end{aligned}
$$

for all $\eta_{h} \in S_{h}$ and $\rho_{h} \in K_{h}$.

Lemma 3.1. We have

$$
\begin{gathered}
\sup _{t \in[0, T]}\left|u_{h}(t)\right|_{0}^{2} \leq C \\
\int_{0}^{T}\left(\left|\left[\zeta\left(\varphi_{h}^{-}\right)\right]^{\frac{1}{2}} \nabla u_{h}^{+}\right|_{0}^{2}+\left|\left[\delta\left(\varphi_{h}^{-}\right)\right]^{\frac{1}{2}} u_{h}^{+}\right|_{0}^{2}\right) \mathrm{d} t \leq C,
\end{gathered}
$$

and

$$
\int_{0}^{T}\left|u_{h}^{+}-u_{h}^{-}\right|_{0}^{2} \mathrm{~d} t \leq C \Delta t
$$

Proof. Setting $\eta_{h}=u_{h}^{n+1}$ in 2.2a we obtain from 1.3 and $\varphi_{h}^{n} \in K$ that

$$
\begin{gathered}
\frac{\varepsilon^{2}}{2}\left|u_{h}^{n+1}-u_{h}^{n}\right|_{h}^{2}+\frac{\varepsilon^{2}}{2}\left(\left|u_{h}^{n+1}\right|_{h}^{2}-\left|u_{h}^{n}\right|_{h}^{2}\right)+\Delta t\left|\left[\zeta\left(\varphi_{h}^{n}\right)\right]^{\frac{1}{2}} \nabla u_{h}^{n+1}\right|_{0}^{2}+\frac{\Delta t}{\varepsilon \alpha}\left|\left[\delta\left(\varphi_{h}^{n}\right)\right]^{\frac{1}{2}} u_{h}^{n+1}\right|_{h}^{2} \\
=\frac{Q \Delta t}{\varepsilon}\left(\delta\left(\varphi_{h}^{n}\right), u_{h}^{n+1}\right)_{h}-\Delta t\left(\zeta\left(\varphi_{h}^{n}\right), u_{h}^{n+1}\right)_{h} \leq C \Delta t\left|u_{h}^{n+1}\right|_{h}^{2}
\end{gathered}
$$

Summing over $n=0, \ldots, N-1$, using a discrete Gronwall inequality and recalling (2.3) gives the required results.

Lemma 3.2. We have that

$$
\sup _{t \in[0, T]}\left|\nabla \varphi_{h}(t)\right|_{0}^{2}+\int_{0}^{T}\left|\partial_{t} \varphi_{h}\right|_{0}^{2} \mathrm{~d} t \leq C,
$$

and

$$
\int_{0}^{T}\left|\nabla\left(\varphi_{h}^{+}-\varphi_{h}^{-}\right)\right|_{0}^{2} \mathrm{~d} t \leq C \Delta t
$$

Proof. Choosing $\rho_{h}=\varphi_{h}^{n} \in K_{h}$ in $\left.2.2 \mathrm{~b}\right)$, re-arranging and using the elementary identity

$$
2 r(r-s)=\left(r^{2}-s^{2}\right)+(r-s)^{2} \quad \forall r, s \in \mathbb{R},
$$

gives

$$
\begin{aligned}
\Delta t\left|\frac{\varphi_{h}^{n+1}-\varphi_{h}^{n}}{\Delta t}\right|_{h}^{2} & +\frac{\beta}{2}\left(\left|\nabla \varphi_{h}^{n+1}\right|_{0}^{2}-\left|\nabla \varphi_{h}^{n}\right|_{0}^{2}\right)+\frac{\beta}{2}\left|\nabla\left(\varphi_{h}^{n+1}-\varphi_{h}^{n}\right)\right|_{0}^{2} \\
& +\frac{\beta}{2 \varepsilon^{2}}\left(\left|\varphi_{h}^{n+1}\right|_{h}^{2}-\left|\varphi_{h}^{n}\right|_{h}^{2}\right)+\frac{\beta}{2 \varepsilon^{2}}\left|\varphi_{h}^{n+1}-\varphi_{h}^{n}\right|_{h}^{2} \leq \frac{c_{W}}{\varepsilon \alpha}\left(u_{h}^{n}, \varphi_{h}^{n+1}-\varphi_{h}^{n}\right)_{h} .
\end{aligned}
$$


Applying Young's inequality to the right hand side yields

$$
\begin{aligned}
\frac{\Delta t}{2}\left|\frac{\varphi_{h}^{n+1}-\varphi_{h}^{n}}{\Delta t}\right|_{h}^{2} & +\frac{\beta}{2}\left(\left|\nabla \varphi_{h}^{n+1}\right|_{0}^{2}-\left|\nabla \varphi_{h}^{n}\right|_{0}^{2}\right)+\frac{\beta}{2}\left|\nabla\left(\varphi_{h}^{n+1}-\varphi_{h}^{n}\right)\right|_{0}^{2} \\
& \leq \frac{\Delta t}{2}\left(\frac{c_{W}}{\varepsilon \alpha}\right)^{2}\left|u_{h}^{n}\right|_{h}^{2}+\frac{\beta}{2 \varepsilon^{2}}\left(\left|\varphi_{h}^{n+1}\right|_{h}^{2}-\left|\varphi_{h}^{n}\right|_{h}^{2}\right) .
\end{aligned}
$$

Summing over $n=0, \ldots, N-1$ and noting (2.3), $\varphi_{h}^{n} \in K_{h}$ and (3.2) yields (3.5) and (3.6.).

Lemma 3.3. We have

$$
\int_{0}^{T}\left\|\partial_{t} u_{h}\right\|_{\left(H^{1}(\Omega)\right)^{\prime}}^{2} \mathrm{~d} t \leq C .
$$

Proof. Let $\psi \in H^{1}(\Omega)$ be arbitrary, and let $J_{h} \psi \in S_{h}$ be its $L^{2}$-projection, defined via

$$
\left(\psi, v_{h}\right)=\left(J_{h} \psi, v_{h}\right)_{h} \quad \forall v_{h} \in S_{h}
$$

We have $\left(v_{h}, \psi\right)_{\left(\left(H^{1}\right)^{\prime}, H^{1}\right)}=\left(v_{h}, \psi\right)=\left(v_{h}, J_{h} \psi\right)_{h}$ for $v_{h} \in S_{h}$, and and so setting $\eta_{h}=J_{h} \psi$ in 2.2a yields

$$
\begin{aligned}
\frac{\varepsilon^{2}}{\Delta t}\left(u_{h}^{n+1}-u_{h}^{n}, \psi\right)_{\left(\left(H^{1}\right)^{\prime}, H^{1}\right)}= & -\left(\zeta\left(\varphi_{h}^{n}\right) \nabla u_{h}^{n+1}, \nabla\left(J_{h} \psi\right)\right)-\frac{1}{\varepsilon \alpha}\left(\delta\left(\varphi_{h}^{n}\right) u_{h}^{n+1}, J_{h} \psi\right)_{h} \\
& +\frac{Q}{\varepsilon}\left(\delta\left(\varphi_{h}^{n}\right), J_{h} \psi\right)_{h}-\left(\zeta\left(\varphi_{h}^{n}\right), J_{h} \psi\right)_{h} .
\end{aligned}
$$

It can be shown that $\left\|J_{h} \psi\right\|_{1} \leq C\|\psi\|_{1}$, for all $\psi \in H^{1}(\Omega)$ (see, for example, [1]). Using this fact, together with 3.2 and the bounds on the $L^{\infty}$ norms of $\delta\left(\varphi_{h}^{n}\right)$ and $\zeta\left(\varphi_{h}^{n}\right)$, we have the following for all $\psi \in H^{1}(\Omega)$

$$
\begin{aligned}
\frac{\varepsilon^{2}}{\Delta t}\left(u_{h}^{n+1}-u_{h}^{n}, \psi\right)_{\left(\left(H^{1}\right)^{\prime}, H^{1}\right)} & \leq C\left|\left[\zeta\left(\varphi_{h}^{n}\right)\right]^{\frac{1}{2}} \nabla u_{h}^{n+1}\right|_{0}\left|\nabla\left(J_{h} \psi\right)\right|_{0}+C\left|J_{h} \psi\right|_{0} \\
& \leq C\left(1+\left|\left[\zeta\left(\varphi_{h}^{n}\right)\right]^{\frac{1}{2}} \nabla u_{h}^{n+1}\right|_{0}\right)\|\psi\|_{1}
\end{aligned}
$$

We conclude that

$$
\left\|\frac{u_{h}^{n+1}-u_{h}^{n}}{\Delta t}\right\|_{\left(H^{1}(\Omega)\right)^{\prime}} \leq C\left(1+\left|\left[\zeta\left(\varphi_{h}^{n}\right)\right]^{\frac{1}{2}} \nabla u_{h}^{n+1}\right|_{0}\right) .
$$

Squaring, multiplying by $\Delta t$, and summing from $n=0, \ldots, N-1$ yields the required result, on noting (3.3).

From Lemmas 3.2 and 3.3 and the Aubin-Lions lemma, we have that there exist functions $\varphi \in L^{\infty}\left(0, T ; H^{1}(\Omega)\right) \cap H^{1}\left(0, T ;\left(H^{1}(\Omega)\right)^{\prime}\right), u \in L^{\infty}(\Omega \times(0, T)) \cap H^{1}\left(0, T ;\left(H^{1}(\Omega)\right)^{\prime}\right)$ and 
$F \in L^{2}\left(0, T ;\left[L^{2}(\Omega)\right]^{2}\right)$ such that, after possibly re-indexing from subsequences, it holds as $h \rightarrow 0$ that

$$
\begin{aligned}
\varphi_{h}^{( \pm)} \stackrel{*}{\rightarrow} \varphi & \text { in } L^{\infty}\left(0, T ; H^{1}(\Omega)\right), \\
\partial_{t} \varphi_{h} \rightarrow \partial_{t} \varphi & \text { in } L^{2}\left(0, T ; L^{2}(\Omega)\right), \\
\varphi_{h}^{( \pm)} \rightarrow \varphi & \text { in } L^{2}\left(0, T ; L^{2}(\Omega)\right), \\
u_{h}^{( \pm)} \rightarrow u & \text { in } L^{\infty}\left(0, T ; L^{2}(\Omega)\right), \\
\partial_{t} u_{h} \rightarrow \partial_{t} u & \text { in } L^{2}\left(0, T ;\left(H^{1}(\Omega)\right)^{\prime}\right), \\
\zeta\left(\varphi_{h}^{-}\right) \nabla u_{h}^{+} \rightarrow F & \text { in } L^{2}\left(0, T ;\left[L^{2}(\Omega)\right]^{2}\right) .
\end{aligned}
$$

Here (3.7f) follows directly from $(3.3)$ and the bound on the $L^{\infty}$ norm of $\zeta\left(\varphi_{h}^{-}\right)$. The function $F$ will be identified later, see Lemma 3.8.

Theorem 3.4. The functions $\varphi$ and $u$ in (3.7) satisfy (2.1b).

Proof. Using (3.7a $-3.7 \mathrm{~d})$ we show that $2.2 \mathrm{~b}$ ) converges to $2.1 \mathrm{~b})$. Starting with $2.2 \mathrm{~b}$, we multiply by an arbitrary $\psi \in C_{0}^{\infty}(0, T), \psi \geq 0$, and integrate over $t \in(0, T)$, to obtain

$$
\begin{aligned}
& \underbrace{\frac{\varepsilon}{\Delta t} \int_{0}^{T} \psi\left(\varphi_{h}^{+}-\varphi_{h}^{-}, \rho_{h}-\varphi_{h}^{+}\right)_{h} \mathrm{~d} t}_{(1)}+\underbrace{\varepsilon \beta \int_{0}^{T} \psi\left(\nabla \varphi_{h}^{+}, \nabla\left(\rho_{h}-\varphi_{h}^{+}\right)\right) \mathrm{d} t}_{(2)} \\
& -\underbrace{\frac{\beta}{\varepsilon} \int_{0}^{T} \psi\left(\varphi_{h}^{+}, \rho_{h}-\varphi_{h}^{+}\right)_{h} \mathrm{~d} t}_{(3)}-\underbrace{\frac{c_{W}}{\alpha} \int_{0}^{T} \psi\left(u_{h}^{+}, \rho_{h}-\varphi_{h}^{+}\right)_{h} \mathrm{~d} t}_{(4)} \geq 0 .
\end{aligned}
$$

Since $\rho \in K$, there exists a sequence $\rho_{h} \in K_{h}$ such that $\rho_{h} \rightarrow \rho$ in $H^{1}(\Omega)$ as $h \rightarrow 0$.

For all but the second integral we use the well known inequality,

$$
\left|\left(\eta_{h}, \chi\right)-\left(\eta_{h}, \chi\right)_{h}\right| \leq C h\left|\eta_{h}\right|_{1}|\chi|_{0} \quad \forall \eta_{h}, \chi \in S_{h} .
$$

For (1) we note that $\partial_{t} \varphi_{h}=\frac{\varphi_{h}^{+}-\varphi_{h}^{-}}{\Delta t}$ on $\left(t_{n+1}, t_{n}\right)$, and we can thus apply $3.7 \mathrm{~b}$ ) and $\left.3.7 \mathrm{c}\right)$. For (2) we use (3.7a), (3.7c), and the weak lower semi-continuity of the $L^{2}$ norm (which gives that $-|\nabla \varphi|_{2}^{2} \geq-\liminf _{h \rightarrow 0}\left|\nabla \varphi_{h}^{+}\right|_{2}^{2}$ ). For (3) we use (3.7c). Finally, for (4), we use (3.7c) and $3.7 \mathrm{~d}$ ). This yields

$$
\begin{gathered}
\varepsilon \int_{0}^{T} \psi\left(\partial_{t} \varphi, \rho-\varphi\right) \mathrm{d} t+\varepsilon \beta \int_{0}^{T} \psi(\nabla \varphi, \nabla(\rho-\varphi)) \mathrm{d} t-\frac{\beta}{\varepsilon} \int_{0}^{T} \psi(\varphi, \rho-\varphi) \mathrm{d} t \\
-\frac{c_{W}}{\alpha} \int_{0}^{T} \psi(u, \rho-\varphi) \mathrm{d} t \geq 0 .
\end{gathered}
$$

As $\psi \geq 0$ is arbitrary, this gives us the result in the limit as $h \rightarrow 0$.

Lemma 3.5. We have

$$
\begin{aligned}
\zeta\left(\varphi_{h}^{( \pm)}\right) \rightarrow \zeta(\varphi) & \text { in } L^{2}\left(0, T ; L^{2}(\Omega)\right), \\
\delta\left(\varphi_{h}^{( \pm)}\right) \rightarrow \delta(\varphi) & \text { in } L^{2}\left(0, T ; L^{2}(\Omega)\right), \\
\delta\left(\varphi_{h}^{(-)}\right) u_{h}^{(+)} \rightarrow \delta(\varphi) u & \text { in } L^{2}\left(0, T ; L^{2}(\Omega)\right) .
\end{aligned}
$$


Proof. The statements (3.9a) and $3.9 \mathrm{~b}$ follow trivially from $\varphi_{h} \in K$ and $\left.3.7 \mathrm{c}\right)$. Then (3.9c) follows by combining (3.7d) and (3.9b).

The following lemma provides us the necessary $L^{\infty}\left(0, T ; L^{\infty}(\Omega)\right)$ bound on $u_{h}^{n+1}$ that we require to prove the convergence results in the subsequent lemma, Lemma 3.7 .

Lemma 3.6. Let $\left(u_{h}^{n}, \varphi_{h}^{n}\right) \in S_{h} \times K_{h}$ and let $u_{h}^{n+1} \in S_{h}$ be the unique solution to 2.2a). Then it holds that

$$
-\left|u_{h}^{0}\right|_{0, \infty}-\varepsilon^{-2} T \leq u_{h}^{n+1} \leq \max \left(\alpha Q,\left|u_{h}^{0}\right|_{0, \infty}\right) \quad \text { in } \bar{\Omega} .
$$

Proof. Throughout this proof, we use the shorthand notations $u_{j}^{n+1}=u_{h}^{n+1}\left(p_{j}\right)$, and $\varphi_{j}^{n}=\varphi_{h}^{n}\left(p_{j}\right)$ for $j \in J$. We first use an inductive argument to prove the maximum bound. We assume that $\max _{j \in J} u_{j}^{n} \leq \max \left(\alpha Q,\left|u_{h}^{0}\right|_{0, \infty}\right)$, which clearly holds for $n=0$, and set $u_{j_{0}}^{n+1}:=\max _{j \in J} u_{j}^{n+1}=\max _{\bar{\Omega}} u_{h}^{n+1}$. Since $\mathcal{T}_{h}$ is acute, we have $\left.\left.\nabla \chi_{i}\right|_{\sigma} \cdot \nabla \chi_{j}\right|_{\sigma} \leq 0$ for $i \neq j$, and hence

$$
\begin{aligned}
\left(\zeta\left(\varphi_{h}^{n}\right) \nabla u_{h}^{n+1}, \nabla \chi_{j_{0}}\right) & =\left.\left.\sum_{\sigma \in \mathcal{T}_{h}} \sum_{i \in J} u_{i}^{n+1} \nabla \chi_{i}\right|_{\sigma} \cdot \nabla \chi_{j_{0}}\right|_{\sigma} \int_{\sigma} \zeta\left(\varphi_{h}^{n}\right) \mathrm{d} x \\
& \geq\left.\left.\sum_{\sigma \in \mathcal{T}_{h}} u_{j_{0}}^{n+1} \sum_{i \in J} \nabla \chi_{i}\right|_{\sigma} \cdot \nabla \chi_{j_{0}}\right|_{\sigma} \int_{\sigma} \zeta\left(\varphi_{h}^{n}\right) \mathrm{d} x=0 .
\end{aligned}
$$

Hence choosing $\eta_{h}=\chi_{j_{0}}$ in 2.2a implies

$$
\begin{aligned}
\frac{\varepsilon^{2}}{\Delta t}\left(u_{j_{0}}^{n+1}-u_{j_{0}}^{n}\right)\left(1, \chi_{j_{0}}\right) & \leq\left(\frac{1}{\varepsilon} \delta\left(\varphi_{j_{0}}^{n}\right)\left(Q-\frac{u_{j_{0}}^{n+1}}{\alpha}\right)-\zeta\left(\varphi_{j_{0}}^{n}\right)\right)\left(1, \chi_{j_{0}}\right) \\
& \leq \frac{1}{\varepsilon} \delta\left(\varphi_{j_{0}}^{n}\right)\left(Q-\frac{u_{j_{0}}^{n+1}}{\alpha}\right)\left(1, \chi_{j_{0}}\right) .
\end{aligned}
$$

If $u_{j_{0}}^{n+1}>\max \left(\alpha Q,\left|u_{h}^{0}\right|_{0, \infty}\right)$, then we have $u_{j_{0}}^{n+1}<u_{j_{0}}^{n} \leq \max \left(\alpha Q,\left|u_{h}^{0}\right|_{0, \infty}\right)$, which is a contradiction, and thus $u_{j_{0}}^{n+1} \leq \max \left(\alpha Q,\left|u_{h}^{0}\right|_{0, \infty}\right)$.

For the minimum bound, we again use an inductive argument. We assume that $\min _{j \in J} u_{j}^{n} \geq-\frac{n \Delta t}{\varepsilon^{2}}-\left|u_{h}^{0}\right|_{0, \infty}$ and set $u_{j_{0}}^{n+1}:=\min _{j \in J} u_{j}^{n+1}$. Choosing $\eta_{h}=\chi_{j_{0}}$ in 2.2a) yields

$$
\frac{\varepsilon^{2}}{\Delta t}\left(u_{j_{0}}^{n+1}-u_{j_{0}}^{n}\right)\left(1, \chi_{j_{0}}\right) \geq\left(\frac{1}{\varepsilon} \delta\left(\varphi_{j_{0}}^{n}\right)\left(Q-\frac{u_{j_{0}}^{n+1}}{\alpha}\right)-\zeta\left(\varphi_{j_{0}}^{n}\right)\right)\left(1, \chi_{j_{0}}\right),
$$

where we used a similar argument to 3.10 . If $u_{j_{0}}^{n+1} \geq 0$ we have nothing to prove, and so it is sufficient to consider the case $u_{j_{0}}^{n+1}<0$. Then $Q-\frac{u_{j_{0}}^{n+1}}{\alpha}>0$, which together with (3.11) and (1.3) implies that

$$
\frac{\varepsilon^{2}}{\Delta t}\left(u_{j_{0}}^{n+1}-u_{j_{0}}^{n}\right) \geq-\zeta\left(\varphi_{j_{0}}^{n}\right) \geq-1
$$

Hence we have that

$$
u_{j_{0}}^{n+1} \geq-\frac{\Delta t}{\varepsilon^{2}}+u_{j_{0}}^{n} \geq-\frac{(n+1) \Delta t}{\varepsilon^{2}}-\left|u_{h}^{0}\right|_{0, \infty}
$$

which proves the inductive assumption and hence yields the desired lower bound. 
Lemma 3.7. We have $\delta(\varphi) u, \zeta(\varphi) u \in L^{2}\left(0, T ; H^{1}(\Omega)\right)$ and

$$
\begin{aligned}
& \delta\left(\varphi_{h}\right) u_{h} \rightarrow \delta(\varphi) u \quad \text { in } L^{2}\left(0, T ; L^{2}(\Omega)\right), \\
& \zeta\left(\varphi_{h}\right) u_{h} \rightarrow \zeta(\varphi) u \quad \text { in } L^{2}\left(0, T ; L^{2}(\Omega)\right) .
\end{aligned}
$$

Proof. We first obtain a bound on $\delta\left(\varphi_{h}\right) u_{h}$ in $L^{2}\left(0, T ; H^{1}(\Omega)\right)$ and then we obtain a bound on $\partial_{t}\left(\delta\left(\varphi_{h}\right) u_{h}\right)$ in $L^{2}\left(0, T ;\left(W^{1, p}(\Omega)\right)^{\prime}\right)$, for $p \in(2, \infty)$. To bound $\left|\nabla\left(\delta\left(\varphi_{h}\right) u_{h}\right)\right|_{0}^{2}$ we first note that from Lemma 3.2 we have $\left|\nabla \delta\left(\varphi_{h}\right)\right|_{0}^{2} \leq C$, and thus noting Lemma 3.6, we have

$$
\left|\nabla\left(\delta\left(\varphi_{h}\right) u_{h}\right)\right|_{0}^{2} \leq\left|\nabla \delta\left(\varphi_{h}\right)\right|_{0}^{2}\left|u_{h}\right|_{0, \infty}^{2}+\left|\delta\left(\varphi_{h}\right) \nabla u_{h}\right|_{0}^{2} \leq C+\left|\delta\left(\varphi_{h}\right) \nabla u_{h}\right|_{0}^{2}
$$

Noting that, for $t \in\left(t_{n}, t_{n+1}\right)$,

$$
\delta\left(\varphi_{h}\right) \leq \delta\left(\varphi_{h}^{n}\right)+C\left|\varphi_{h}^{n+1}-\varphi_{h}^{n}\right|,
$$

and using the bound on the $L^{\infty}$ norm of $\delta\left(\varphi_{h}\right)$, we have, for $t \in\left(t_{n}, t_{n+1}\right)$,

$$
\begin{aligned}
\left|\delta\left(\varphi_{h}\right) \nabla u_{h}\right|_{0}^{2} & \leq C\left|\delta\left(\varphi_{h}^{n}\right) \nabla u_{h}\right|_{0}^{2}+C\left|\left(\varphi_{h}^{n+1}-\varphi_{h}^{n}\right) \nabla u_{h}\right|_{0}^{2} \\
& \leq C\left|\delta\left(\varphi_{h}^{n}\right) \nabla u_{h}^{n+1}\right|_{0}^{2}+C\left|\delta\left(\varphi_{h}^{n}\right) \nabla\left(u_{h}^{n+1}-u_{h}^{n}\right)\right|_{0}^{2}+C\left|\left(\varphi_{h}^{n+1}-\varphi_{h}^{n}\right) \nabla u_{h}\right|_{0}^{2} \\
& \leq C\left|\left[\delta\left(\varphi_{h}^{n}\right)\right]^{\frac{1}{2}} \nabla u_{h}^{n+1}\right|_{0}^{2}+C\left|\nabla\left(u_{h}^{n+1}-u_{h}^{n}\right)\right|_{0}^{2}+C\left|\nabla u_{h}\right|_{0, \infty}^{2}\left|\varphi_{h}^{n+1}-\varphi_{h}^{n}\right|_{0}^{2}, \\
& \leq C\left|\left[\delta\left(\varphi_{h}^{n}\right)\right]^{\frac{1}{2}} \nabla u_{h}^{n+1}\right|_{0}^{2}+C h^{-2}\left(\left|u_{h}^{n+1}-u_{h}^{n}\right|_{0}^{2}+\left|u_{h}\right|_{0, \infty}^{2}\left|\varphi_{h}^{n+1}-\varphi_{h}^{n}\right|_{0}^{2}\right) \\
& \leq C\left|\left[\zeta\left(\varphi_{h}^{n}\right)\right]^{\frac{1}{2}} \nabla u_{h}^{n+1}\right|_{0}^{2}+C h^{-2}\left(\left|u_{h}^{n+1}-u_{h}^{n}\right|_{0}^{2}+\left|\varphi_{h}^{n+1}-\varphi_{h}^{n}\right|_{0}^{2}\right),
\end{aligned}
$$

where we have used the fact that $\delta(s)=\frac{2}{\pi}(1+s)(1-s)=\frac{4}{\pi}(1-s) \zeta(s)$, recall (1.3) and, noting that we are restricting ourselves to $\mathbb{R}^{2}$, the inverse estimate

$$
\left|\nabla v_{h}\right|_{0, p} \leq C h^{-1}\left|v_{h}\right|_{0, p} \quad v_{h} \in S_{h}, \text { for } p \in[1, \infty] .
$$

Summing over $n$, multiplying by $\Delta t$ and recalling that $\Delta t \leq C h^{2}$ yields, in light of the bounds from Lemmas 3.2 and 3.1 , that

$$
\begin{aligned}
\int_{0}^{T}\left|\delta\left(\varphi_{h}\right) \nabla u_{h}\right|_{0}^{2} \mathrm{~d} t \leq & C \int_{0}^{T}\left|\left[\zeta\left(\varphi_{h}^{-}\right)\right]^{\frac{1}{2}} \nabla u_{h}^{+}\right|_{0}^{2} \mathrm{~d} t+C h^{-2} \int_{0}^{T}\left|u_{h}^{+}-u_{h}^{-}\right|_{0}^{2} \mathrm{~d} t \\
& +C h^{-2} \int_{0}^{T}\left|\varphi_{h}^{+}-\varphi_{h}^{-}\right|_{0}^{2} \mathrm{~d} t \leq C+C h^{-2} \Delta t \leq C .
\end{aligned}
$$

In what follows, we fix $p>2$, and bound

$$
\int_{0}^{T}\left\|\partial_{t}\left(\delta\left(\varphi_{h}\right) u_{h}\right)\right\|_{\left(W^{1, p}(\Omega)\right)^{\prime}}^{2} \mathrm{~d} t
$$

Let $\psi \in W^{1, p}(\Omega)$ be arbitrary. Then, noting Lemma 3.6, we have

$$
\begin{aligned}
\left(\partial_{t}\left(\delta\left(\varphi_{h}\right) u_{h}\right), \psi\right. & { }_{\left(\left(W^{1, p}\right)^{\prime}, W^{1, p}\right)}=\left(\partial_{t}\left(\delta\left(\varphi_{h}\right) u_{h}\right), \psi\right) \leq C\left|\left(\varphi_{h} \partial_{t} \varphi_{h} u_{h}, \psi\right)\right|+C\left|\left(\delta\left(\varphi_{h}\right) \partial_{t} u_{h}, \psi\right)\right| \\
& \leq C|\psi|_{0}\left|\partial_{t} \varphi_{h}\right|_{0}+\left\|\partial_{t} u_{h}\right\|_{\left(H^{1}(\Omega)\right)^{\prime}}\left\|\psi \delta\left(\varphi_{h}\right)\right\|_{1} \\
& \leq C|\psi|_{0}\left|\partial_{t} \varphi_{h}\right|_{0}+\left\|\partial_{t} u_{h}\right\|_{\left(H^{1}(\Omega)\right)^{\prime}}\left(\|\psi\|_{1}+\|\psi\|_{0, \infty}\left\|\delta\left(\varphi_{h}\right)\right\|_{1}\right) \\
& \leq C\|\psi\|_{1, p}\left(\left|\partial_{t} \varphi_{h}\right|_{0}+\left\|\partial_{t} u_{h}\right\|_{\left(H^{1}(\Omega)\right)^{\prime}}\left(1+\left\|\varphi_{h}\right\|_{1}\right)\right) .
\end{aligned}
$$


Using bounds from Lemmas 3.2 and 3.3 , and the continuous embedding $W^{1, p}(\Omega) \hookrightarrow$ $C^{0}(\bar{\Omega})$, we deduce a bound on (3.14). This, combined with the bound $\left|\nabla\left(\delta\left(\varphi_{h}\right) u_{h}\right)\right|_{0}^{2} \leq C$ and a well-known compactness results yields the desired strong convergence result (3.12a). The proof for $\zeta(\varphi) u$ and $3.12 \mathrm{~b}$ is analogous.

We define the set

$$
U:=\{(x, t) \in \Omega \times(0, T) \mid \zeta(\varphi(x, t))>0\} .
$$

As $u \in L^{\infty}(\Omega \times(0, T))$, the regularity theory for parabolic variational inequalities, see [13, gives

$$
\varphi \in L^{p}\left(0, T ; W^{2, p}(\Omega)\right), \quad \text { and } \quad \partial_{t} \varphi \in L^{p}\left(0, T ; L^{p}(\Omega)\right), \quad \text { for } 1 \leq p<\infty .
$$

Thus, by the Aubin-Lions lemma, $\varphi \in C^{0}(\overline{\Omega \times(0, T)})$, and hence the set $U$ is open.

Lemma 3.8. The function $F$ in (3.7f) satisfies $F=\chi(U) \zeta(\varphi) \nabla u$ almost everywhere in $\Omega \times(0, T)$, where $\chi(U)$ is the characteristic function of $U$.

Proof. In order to identify $F$ on $U$ we show that $\left(\zeta(\varphi)^{2} u\right)_{x_{i}} \in L^{2}\left(0, T ; L^{2}(\Omega)\right)$ and

$$
\left(\zeta(\varphi)^{2} u\right)_{x_{i}}=\zeta(\varphi) u \varphi_{x_{i}}+F_{i} \zeta(\varphi), i=1,2
$$

By Lemma 3.7 we have $\zeta\left(\varphi_{h}\right) u_{h} \rightarrow \zeta(\varphi) u$ in $L^{2}\left(0, T ; L^{2}(\Omega)\right)$, so that

$$
\int_{0}^{T}\left(\zeta(\varphi)^{2} u, \psi_{x_{i}}\right) \mathrm{d} t=\lim _{h \rightarrow 0} \int_{0}^{T}\left(\zeta\left(\varphi_{h}\right)^{2} u_{h}, \psi_{x_{i}}\right) \mathrm{d} t, \quad \forall \psi \in C_{0}^{\infty}(\Omega \times(0, T)) .
$$

Using integration by parts on the right hand side integral we have

$$
\begin{aligned}
\int_{0}^{T}\left(\zeta\left(\varphi_{h}\right)^{2} u_{h}, \psi_{x_{i}}\right) \mathrm{d} t & =-\int_{0}^{T}\left(\zeta\left(\varphi_{h}\right)^{2} u_{h, x_{i}}, \psi\right) \mathrm{d} t-\int_{0}^{T}\left(\left(\zeta\left(\varphi_{h}\right)^{2}\right)_{x_{i}} u_{h}, \psi\right) \mathrm{d} t \\
& =-\int_{0}^{T}\left(\zeta\left(\varphi_{h}\right)\left(\zeta\left(\varphi_{h}\right) u_{h, x_{i}}\right), \psi\right) \mathrm{d} t-\int_{0}^{T}\left(\zeta\left(\varphi_{h}\right) u_{h} \varphi_{h, x_{i}}, \psi\right) \mathrm{d} t .
\end{aligned}
$$

Since $\zeta\left(\varphi_{h}\right) u_{h} \rightarrow \zeta(\varphi) u$ in $L^{2}\left(0, T ; L^{2}(\Omega)\right)$ (by Lemma 3.7), the dominated convergence theorem implies that $\zeta\left(\varphi_{h}\right) u_{h} \psi \rightarrow \zeta(\varphi) u \psi$ in $L^{2}\left(0, T ; L^{2}(\Omega)\right)$. Using this, and $\nabla \varphi_{h} \rightarrow \nabla \varphi$ in $L^{2}\left(0, T ;\left[L^{2}(\Omega)\right]^{2}\right)$ from $(3.7 \mathrm{a})$, we have

$$
\int_{0}^{T}\left(\zeta\left(\varphi_{h}\right) u_{h} \varphi_{h, x_{i}}, \psi\right) \mathrm{d} t \rightarrow \int_{0}^{T}\left(\zeta(\varphi) u \varphi_{x_{i}}, \psi\right) \mathrm{d} t, \quad \forall \psi \in C_{0}^{\infty}(\Omega \times(0, T)) .
$$

By (3.7f) and 3.9a we have

$$
\int_{0}^{T}\left(\zeta\left(\varphi_{h}\right)\left(\zeta\left(\varphi_{h}\right) u_{h, x_{i}}\right), \psi\right) \mathrm{d} t \rightarrow \int_{0}^{T}\left(\zeta(\varphi) F_{i}, \psi\right) \mathrm{d} t, \quad \forall \psi \in C_{0}^{\infty}(\Omega \times(0, T)) .
$$

Thus, as $\psi$ is arbitrary, we have (3.15) almost everywhere. 
We now identify $F$ on $U$. Let $\psi \in C_{0}^{\infty}(U)$ be arbitrary. Using integration by parts we have

$$
\begin{aligned}
-\int_{U} u_{x_{i}} \psi \mathrm{d} x \mathrm{~d} t=\int_{U} u \psi_{x_{i}} \mathrm{~d} x \mathrm{~d} t & =\int_{U} \zeta(\varphi)^{2} u \frac{1}{\zeta(\varphi)^{2}} \psi_{x_{i}} \mathrm{~d} x \mathrm{~d} t \\
& =-\int_{U}\left(\zeta(\varphi)^{2} u\right)_{x_{i}} \frac{\psi}{\zeta(\varphi)^{2}} \mathrm{~d} x \mathrm{~d} t+\int_{U} \frac{1}{\zeta(\varphi)} u \varphi_{x_{i}} \psi \mathrm{d} x \mathrm{~d} t .
\end{aligned}
$$

Substituting in (3.15), we have

$$
-\int_{U} u_{x_{i}} \psi \mathrm{d} x \mathrm{~d} t=-\int_{U} F_{i} \frac{1}{\zeta(\varphi)} \psi \mathrm{d} x \mathrm{~d} t
$$

Since $\psi$ is arbitrary, this gives us that $u_{x_{i}} \zeta(\varphi)=F_{i}$ almost everywhere on $U$.

It remains to identify $F$ on $U^{c}:=\Omega \times(0, T) \backslash U$. Let $\psi \in C_{0}^{\infty}(\Omega \times(0, T))$ be arbitrary. We use that $1-\chi(U)=0$ on $U$ to give

$$
\begin{aligned}
& \left|\int_{U^{c}} \zeta\left(\varphi_{h}^{-}\right) u_{h, x_{i}}^{+} \psi \mathrm{d} x \mathrm{~d} t\right|=\left|\int_{0}^{T} \int_{\Omega} \zeta\left(\varphi_{h}^{-}\right) u_{h, x_{i}}^{+}(1-\chi(U)) \psi \mathrm{d} x \mathrm{~d} t\right| \\
& \leq\left(\int_{0}^{T} \int_{\Omega} \zeta\left(\varphi_{h}^{-}\right)\left|\nabla u_{h}^{+}\right|^{2} \mathrm{~d} x \mathrm{~d} t\right)^{\frac{1}{2}}\left(\int_{0}^{T} \int_{\Omega} \zeta\left(\varphi_{h}^{-}\right)(1-\chi(U))^{2} \psi^{2} \mathrm{~d} x \mathrm{~d} t\right)^{\frac{1}{2}}, \\
& \leq C\left(\int_{0}^{T} \int_{\Omega} \zeta\left(\varphi_{h}^{-}\right)(1-\chi(U))^{2} \psi^{2} \mathrm{~d} x \mathrm{~d} t\right)^{\frac{1}{2}}
\end{aligned}
$$

where we have recalled $(3.3)$. By $3.9 \mathrm{a}$

$$
\left(\int_{0}^{T} \int_{\Omega} \zeta\left(\varphi_{h}^{-}\right)(1-\chi(U))^{2} \psi^{2} \mathrm{~d} x \mathrm{~d} t\right)^{\frac{1}{2}} \rightarrow 0 .
$$

Thus, from (3.16), we have

$$
\int_{U^{c}} \zeta\left(\varphi_{h}^{-}\right) u_{h, x_{i}}^{+} \psi \mathrm{d} x \mathrm{~d} t \rightarrow 0
$$

Recalling (3.7f), we have

$$
\int_{0}^{T} \int_{\Omega} \zeta\left(\varphi_{h}^{-}\right) u_{h, x_{i}}^{+} \psi \mathrm{d} x \mathrm{~d} t \rightarrow \int_{0}^{T} \int_{\Omega} F_{i} \psi \mathrm{d} x \mathrm{~d} t .
$$

Thus we conclude that $F_{i}=0$ almost everywhere in $U^{c}, i=1,2$.

Theorem 3.9. The functions $\varphi$ and $u$ in (3.7) satisfy

$$
\begin{aligned}
\varepsilon^{2} \int_{0}^{T} \psi\left(\partial_{t} u, \eta\right)_{\left(\left(H^{1}\right)^{\prime}, H^{1}\right)} \mathrm{d} t & +\int_{0}^{T} \psi \int_{\{\zeta>0\}} \zeta(\varphi) \nabla u \cdot \nabla \eta \mathrm{d} x \mathrm{~d} t+\frac{1}{\varepsilon \alpha} \int_{0}^{T} \psi(\delta(\varphi) u, \eta) \mathrm{d} t \\
& =\frac{Q}{\varepsilon} \int_{0}^{T} \psi(\delta(\varphi), \eta) \mathrm{d} t-\int_{0}^{T} \psi(\zeta(\varphi), \eta) \mathrm{d} t
\end{aligned}
$$

for an arbitrary $\eta \in H^{1}(\Omega)$ and $\psi \in C_{0}^{\infty}(0, T)$. 
Proof. Choosing arbitrary functions $\eta \in H^{1}(\Omega)$ and $\psi \in C_{0}^{\infty}(0, T)$, we know that there exists a sequence $\left(\eta_{h}\right) \subset S_{h}$ such that $\eta_{h} \rightarrow \eta$ in $H^{1}(\Omega)$ as $h \rightarrow 0$. Multiplying (3.1a) by $\psi$, and integrating over $t$ gives

$$
\begin{aligned}
& \underbrace{\varepsilon^{2} \int_{0}^{T} \psi\left(\partial_{t} u_{h}, \eta_{h}\right)_{h} \mathrm{~d} t}_{(1)}+\underbrace{\int_{0}^{T} \psi\left(\zeta\left(\varphi_{h}^{-}\right) \nabla u_{h}^{+}, \nabla \eta_{h}\right) \mathrm{d} t}_{(2)}+\underbrace{\frac{1}{\varepsilon \alpha} \int_{0}^{T} \psi\left(\delta\left(\varphi_{h}^{-}\right) u_{h}^{+}, \eta_{h}\right)_{h} \mathrm{~d} t}_{(3)} \\
& =\underbrace{\frac{Q}{\varepsilon} \int_{0}^{T} \psi\left(\delta\left(\varphi_{h}^{-}\right), \eta_{h}\right)_{h} \mathrm{~d} t}_{(4)}-\underbrace{\int_{0}^{T} \psi\left(\zeta\left(\varphi_{h}^{-}\right), \eta_{h}\right)_{h} \mathrm{~d} t}_{(5)} .
\end{aligned}
$$

For all but (2) we use the well known inequality (3.8). For (1) we use (3.7e). For (2) we use (3.7f) and Lemma 3.8. For (3) we use (3.9c). For (4) we use (3.9b). For (5) we use (3.9a).

Remark 3.10. Due to the inverse inequality (3.13) that is used in the proof of Lemma 3.7, our proof of the convergence result in Theorem 3.9 does not extend to $\mathbb{R}^{3}$.

\section{Numerical results}

In this section we display some computational simulations of tumour growth. In all computations we use the finite element approximation $\mathbb{P}_{h}$ that results from taking $\vartheta=1$ in (1.2). We use the finite element toolbox Alberta 2.0, [20], to implement our approximation (2.2). In order to increase the efficiency of the computations, we employ the mesh refinement strategy presented in [12, which gives rise to a fine mesh in the interfacial region where $\left|\varphi_{h}^{n}\right|<1$, a coarse mesh exterior to the tumour where $\varphi_{h}^{n}=-1$ and a standard sized mesh in the interior of the tumour where $\varphi_{h}^{n}=1$, for more details on a similar mesh refinement strategy see [2]. The result of this refinement strategy can be seen in Figure 1, which displays enlarged sections of the computational meshes associated with the simulations presented in Figure 2 at $t=45$ (left) and Figure 3 at $t=7$ (right). We denote the maximum diameter of the triangles in the three meshes by $h_{\text {max }, f}=\max _{\sigma \in \mathcal{T}_{f}^{n}} h_{\sigma}, h_{\text {max }, m}=\max _{\sigma \in \mathcal{T}_{m}^{n}} h_{\sigma}$ and $h_{\text {max }, c}=\max _{\sigma \in \mathcal{T}_{c}^{n}} h_{\sigma}$, where

$$
\mathcal{T}_{f}^{n}:=\left\{\sigma \in \mathcal{T}_{h}|| \varphi_{h}^{n}(x) \mid<0.99 \forall x \in \sigma\right\}, \quad \mathcal{T}_{m}^{n}:=\left\{\sigma \in \mathcal{T}_{h} \mid \varphi_{h}^{n}(x)=1 \forall x \in \sigma\right\},
$$

and

$$
\mathcal{T}_{c}^{n}:=\left\{\sigma \in \mathcal{T}_{h} \mid \varphi_{h}^{n}(x)=-1 \forall x \in \sigma\right\} .
$$

In all our computations we fix $h_{\max , f} \leq h_{\max , m} \leq h_{\max , c}$ with $h_{\max , m} / h_{\max , f} \leq 2^{4}$ and $h_{\max , c} / h_{\max , m} \leq 2^{7}$. The linear system resulting from $2.2 \mathrm{a}$ was solved using a preconditioned conjugated gradient solver with diagonal preconditioning, while a projected SOR method, see [11], was used to solve the system resulting from the variational inequality (2.2b). The results are visualised in Paraview [19]. 

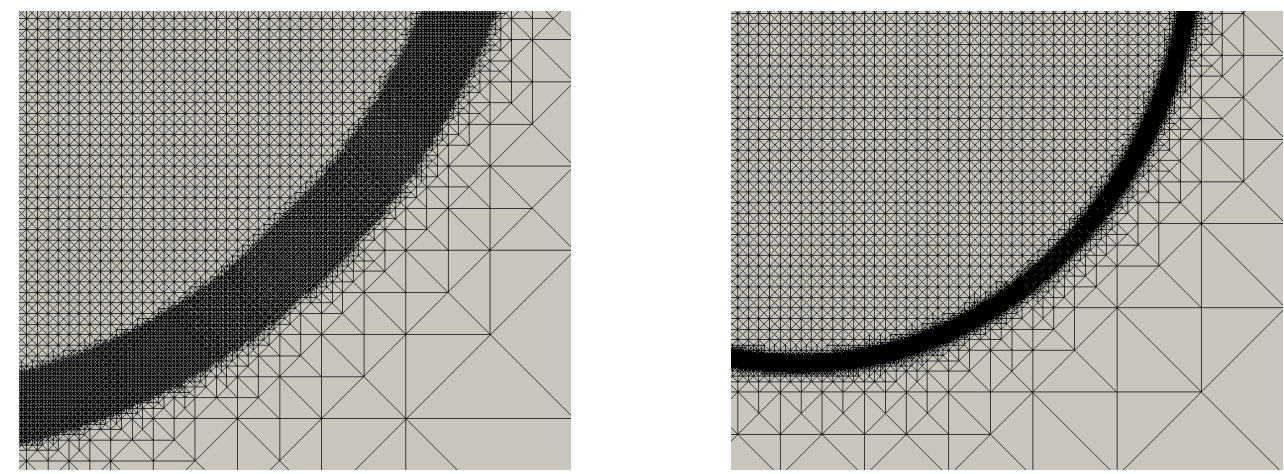

Figure 1: Enlarged sections of the meshes associated with the results in Figure 2 at $t=45$ (left) and Figure 3 at $t=7$ (right).

Noting Lemma 2.1, in all simulations we take $\Delta t<\frac{\varepsilon^{2}}{\beta}$. In addition we set $h_{\text {max }, c} \approx 2.5$ and $h_{\text {max }, m} \approx 0.02$ and we take the the initial pressure to be $u_{0}(x)=0$ for all $x \in \Omega$. Unless otherwise stated we take the initial geometry $\Gamma(0)$ to be an ellipse with length 0.5 and height 1 , that is we choose the initial profile $\varphi_{0}$ as

$$
\varphi_{0}(x)= \begin{cases}1 & r(x) \geq \frac{\varepsilon \pi}{2} \\ \sin \frac{r(x)}{\varepsilon} & -\frac{\varepsilon \pi}{2}<r(x)<\frac{\varepsilon \pi}{2}, \\ -1 & r(x) \leq-\frac{\varepsilon \pi}{2},\end{cases}
$$

where $r(x)=1-\sqrt{4 x_{1}^{2}+x_{2}^{2}}$. The values of $Q, \alpha, \beta, \varepsilon, \Delta t$ and $h_{\text {max }, f}$ used in all our simulations are stated in Table 1. Due to the symmetry of the problems we consider, in all simulations we only solve in the positive quadrant and apply homogeneous Neumann boundary conditions on the boundaries $x_{i}=0, i=1,2$.

\begin{tabular}{|c|c|c|c|c|c|c|}
\hline & $Q$ & $\alpha$ & $\beta$ & $\varepsilon$ & $\Delta t$ & $h_{\max , f}$ \\
\hline Figure 2 & 1 & 1 & 0.1 & 0.04 & $10^{-3}$ & 0.0048 \\
\hline Figure 3 & 1 & 0.1 & 0.1 & 0.01 & $10^{-4}$ & 0.0024 \\
\hline Figure 4 & $\{0.5,0.75,1.5\}$ & 1 & 0.1 & 0.04 & $10^{-3}$ & 0.0048 \\
\hline Figure 5 & 1 & $\{0.5,2,7\}$ & 0.1 & 0.04 & $10^{-3}$ & 0.0048 \\
\hline Figure 6 & 1 & 1 & $\{0.1,0.2,0.5\}$ & 0.04 & $10^{-3}$ & 0.0048 \\
\hline
\end{tabular}

Table 1: Parameters values for Figures 2-6.

\subsection{Radially symmetric solutions}

In the first set of experiments we investigate the accuracy of the numerical scheme as: (i) $\Delta t$ varies, (ii) $\varepsilon$ is reduced, (iii) $\beta$ is increased. To this end, we consider radially symmetric 
solutions by setting $\Gamma(t) \subset \mathbb{R}^{2}$ to be a circle with radius $R(t)$, and we express $u$ in polar coordinates such that $u(r, \theta)=u(r)$. In this setting (1.1) reduces to

$$
\begin{gathered}
u(r)=\frac{1}{4} r^{2}+\alpha Q-\frac{\alpha}{2} R-\frac{1}{4} R^{2}, \\
R^{\prime}(t)=-\frac{\beta}{R}+\frac{1}{\alpha} u(r)=-\frac{\beta}{R}+Q-\frac{R}{2}, \quad R(0):=R_{0} .
\end{gathered}
$$

For each of the simulations we compute the following error

$$
\mathcal{E}_{r}:=\sum_{n=0}^{50}\left|R_{h}\left(t_{n}\right)-R\left(t_{n}\right)\right|^{2},
$$

which is the error between the radii, $R_{h}\left(t_{n}\right)$, for $t_{n}=0.01 n$, obtained from the finite element approximation (2.2a), 2.2b) and the radii, $R\left(t_{n}\right)$, of the corresponding analytical solution, solved using MATLAB's standard solver for ordinary differential equations, ode45.

We first investigate an appropriate choice of $\Delta t$, relative to $h_{\max , f}$. To this end, we set $\alpha=Q=R_{0}=1.0, \beta=0.1$ then, for $\varepsilon=0.02$ and $\varepsilon=0.01$, we consider two values of $h_{\max , f}$ and four values of $\Delta t$. The resulting errors are displayed in Table 2 (for

\begin{tabular}{c|cccc} 
& $\Delta t=0.5 h_{\max , f}$ & $\Delta t=0.2 h_{\max , f}$ & $\Delta t=0.1 h_{\max , f}$ & $\Delta t=0.05 h_{\max , f}$ \\
\hline$h_{\max , f} \approx 0.25 \varepsilon$ & 0.0101793 & 0.0106555 & 0.0107909 & 0.0139744 \\
$h_{\max , f} \approx 0.125 \varepsilon$ & 0.00339958 & 0.00225313 & 0.00200782 & 0.00435587
\end{tabular}

Table 2: $\mathcal{E}_{r}: \varepsilon=0.02$ with $\alpha=Q=R_{0}=1.0, \beta=0.1, \Delta t=0.2 h_{\max , f}$

\begin{tabular}{c|cccc} 
& $\Delta t=0.5 h_{\max , f}$ & $\Delta t=0.2 h_{\max , f}$ & $\Delta t=0.1 h_{\max , f}$ & $\Delta t=0.05 h_{\max , f}$ \\
\hline$h_{\max , f} \approx 0.25 \varepsilon$ & 0.0252947 & 0.0201578 & 0.0184133 & 0.0181777 \\
$h_{\max , f} \approx 0.125 \varepsilon$ & 0.00494710 & 0.00438694 & 0.00434753 & 0.00494933
\end{tabular}

Table 3: $\mathcal{E}_{r}: \varepsilon=0.01$ with $\alpha=Q=R_{0}=1.0, \beta=0.1, \Delta t=0.2 h_{\max , f}$

$\varepsilon=0.02$ ) and Table 3 (for $\varepsilon=0.01$ ). From these tables we see that there is very little difference in the errors for the four values of $\Delta t$, thus for the remaining radially symmetric computations, unless otherwise specified, we set $\Delta t=0.2 h_{\max , f}$.

Remark 4.1. The convergence result in Section 3 relies on the requirement that $\Delta t \leq$ $C h^{2}$, however from Tables 2 and 3 we see that it is sufficient to take $\Delta t \leq C h$ in practice. 


\begin{tabular}{l|cccc} 
& $\varepsilon=0.04$ & $\varepsilon=0.02$ & $\varepsilon=0.01$ & $\varepsilon=0.005$ \\
\hline$\varepsilon \approx 4 h_{\text {max }, f}$ & 0.00929059 & 0.0106555 & 0.0201578 & 0.0304609 \\
$\varepsilon \approx 8 h_{\text {max }, f}$ & 0.0181973 & 0.00225313 & 0.00438694 & 0.008757458
\end{tabular}

Table 4: $\mathcal{E}_{r}: \alpha=Q=R_{0}=1.0, \beta=0.1, \Delta t=0.2 h_{\max , f}$

Next we consider the accuracy of the scheme as $\varepsilon$, and subsequently $h_{\max , f}$ and $\Delta t=$ $0.2 h_{\max , f}$, are reduced. To this end we set $\alpha=Q=R_{0}=1.0, \beta=0.1$ and consider four values of $\varepsilon$. For each value of $\varepsilon$ we consider two values of $h_{\max , f}$. The errors $\mathcal{E}_{r}$ are displayed in Table 4 , from which we see that taking $\varepsilon \approx 4 h_{\max , f}$ yields, for all values of $\varepsilon$, errors that are of the same order of magnitude. Whereas taking $\varepsilon \approx 8 h_{\max , f}$ yields errors that are the same order of magnitude for $\varepsilon=0.02$ and $\varepsilon=0.01$, but gives larger errors for $\varepsilon=0.04$ and $\varepsilon=0.005$.

We conclude our radially symmetric results by considering the accuracy of the scheme when $\beta$ is increased from $\beta=0.1$ to $\beta=1$. In these computations we set $Q=R_{0}=1.5$, $\alpha=1$ and consider two values of $\varepsilon$. We note that increasing $Q$ and $R_{0}$ from 1 to 1.5 ensures growth of the initial circle, recall (4.2). For each value of $\varepsilon$ we consider three values of $h_{\max , f}$. In all computations we set $\Delta t=0.05 h_{\max , f}$, since this results in the restriction $\Delta t<\frac{\varepsilon^{2}}{\beta}$, recall Lemma 2.1. always being satisfied. By comparing Table 5 with Table 4 we see that for $\beta=1$, even when the value of $h_{\max , f}$ is half the size of the values used when $\beta=0.1$, the errors for $\beta=1$ are larger than the errors for $\beta=0.1$. We infer from Tables 4 and 5 that in order to maintain the accuracy of the scheme, a relationship of the form $\varepsilon=\Lambda h_{\max , f}$ should be used, with $\Lambda$ increasing as $\beta$ increases.

\begin{tabular}{l|cc} 
& $\varepsilon=0.04$ & $\varepsilon=0.02$ \\
\hline$\varepsilon \approx 4 h_{\max , f}$ & 0.0891700 & 0.0844617 \\
$\varepsilon \approx 8 h_{\max , f}$ & 0.0468858 & 0.0253982 \\
$\varepsilon \approx 16 h_{\max , f}$ & 0.0350065 & 0.0164192
\end{tabular}

Table 5: $\mathcal{E}_{r}: \beta=1, Q=R_{0}=1.5, \alpha=1, \Delta t=0.05 h_{\max , f}$

\subsection{Comparison with results in [12]}

In the second set of experiments, Figures 2 and 3 , we investigate the effect of using $\vartheta=1$ in (1.2), which we included for the numerical analysis in this paper. We recall that a discretisation of (1.2) with $\theta=0$ was considered in [12], and so we compare our numerical results with those obtained in that paper. In particular, in Figures 2 and 3 we display results that correspond to the ones presented respectively in Figures 10 and 12 of [12]. 
For both simulations we set $\Omega=(-5,5)^{2}, Q=1$ and $\beta=0.1$. The values of $\alpha, \varepsilon, \Delta t$ and $h_{\max , f}$, which differ for the two simulations, are stated in Table 1. For each figure we display plots of the solution $\varphi_{h}^{n}$ in the top row and the pressure $u_{h}^{n}$, restricted to the region in which $\varphi_{h}^{n}>-1$, i.e. the interior of the tumour, in the bottom row. To be consistent with Figures 10 and 12 in [12, the plots in Figure 2 are displayed at $t=0,30,45$ and the plots in Figure 3 are displayed at $t=0,3,7$. For both sets of results we see a close resemblance to the results in [12], with the geometries being visually indistinguishable and only small differences in the values of the discrete pressures. Overall we are satisfied that choosing $\vartheta=1$ in $(1.2)$, compared to $\vartheta=0$, only has a negligible effect on the numerical results for the evolutions we are interested in.

\subsection{The influence of $Q, \alpha$ and $\beta$}

In the remaining figures, Figures $4-6$, we present some parameter studies that investigate the influence of the parameters $Q, \alpha$ and $\beta$, respectively. In all the simulations we set $\Omega=(-10,10)^{2}$ and we display the pressure $u_{h}^{n}$ in the region in which $\varphi_{h}^{n}>-1$. Associated with this parameter study we note that in Section 3.1.2 of [12] a linear stability analysis on a more complicated version of the model (1.1), that includes an additional curvature term on the left hand side of $(1.1 \mathrm{~b})$, yields steady state circular solutions if $3 \alpha \beta>2 Q^{3}$.

Figure 4 displays $u_{h}^{n}$ obtained by setting $\alpha=1, \beta=0.1$ and $Q=0.5$ (top row), $Q=$ 0.75 (middle row) and $Q=1.5$ (bottom row). From this figure we see that for $Q=$ 0.5 the ellipse evolves to form the expected steady state circular solution, since $3 \alpha \beta=$ $0.3>0.25=2 Q^{3}$. For $Q=0.75$ the ellipse extends in the $x_{2}$ direction to produce an elongated geometry that is rounded at both ends. This geometry is reminiscent of the simulation displayed in Figure 8 of [12] that relates to the thin film evolution analysed in the Appendix of [12]. When $Q=1.5$ the ellipse evolves into a more complex geometry, with a rounded and compact structure.

From Figures 2 and 3 we saw the effect that varying $\alpha$ has on the solution, with $\alpha=1$ giving rise to a geometry with four distinct branches, while $\alpha=0.1$ leads to more rounded and compact structure. We investigate the effect of varying $\alpha$ further in Figure 5 in which we set $Q=1$ and $\beta=0.1$. We display $\alpha=0.5$ (top row), $\alpha=2$ (middle row) and $\alpha=7$ (bottom row). From this figure we see that $\alpha=0.5$ gives rise to a rounded and compact structure similar to the one seen in the bottom row of Figure 4 , while for $\alpha=2$ four branches are present at $t=125$ and by $t=170$ these have split and evolved into eight branches. Taking $\alpha=7$ yields the expected steady state circular solution, since we have $3 \alpha \beta=2.1>2=2 Q^{3}$.

Finally, in Figure 6 we see the effect that varying $\beta$ has on the solution. We set $Q=\alpha=1$ and display $\beta=0.5$ (top row) and $\beta=0.2$ (middle row), together with $\beta=0.1$ (bottom row). Here we display $\beta=0.1$ solely for comparison purposes, since it is the same as the solution from Figure 2 except that here $\Omega=(-10,10)^{2}$ rather than $\Omega=(-5,5)^{2}$. In this figure we see that when $\beta=0.5$ the curvature term dominates the motion and the ellipse evolves to a shrinking circle that has almost disappeared by $t=2$. When $\beta=0.2$ an 
elongated geometry with rounded ends is observed that is similar to the one displayed in the middle row of Figure 4.

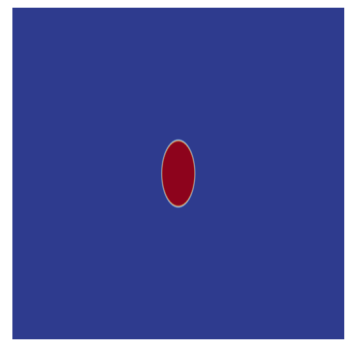

(a) $\mathrm{t}=0$

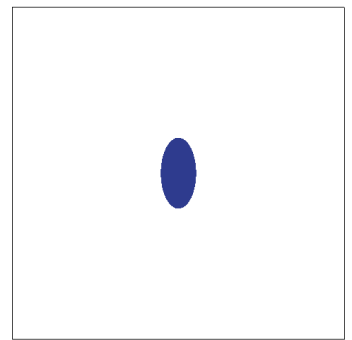

(d) $\mathrm{t}=0$

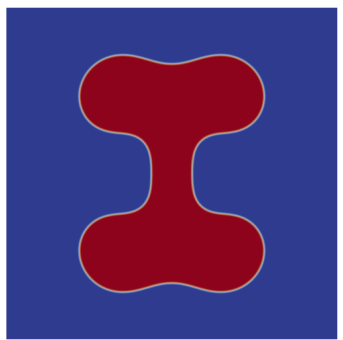

(b) $t=30$

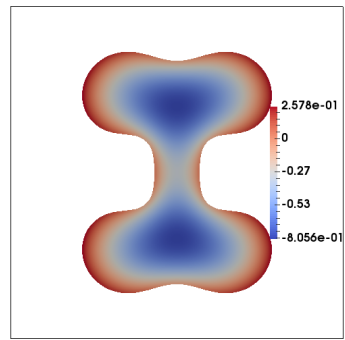

(e) $\mathrm{t}=30$

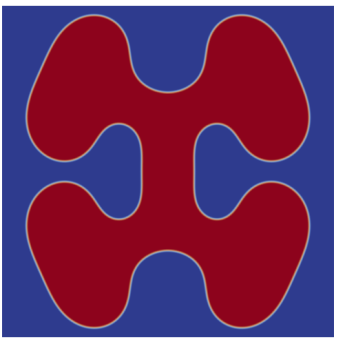

(c) $\mathrm{t}=45$

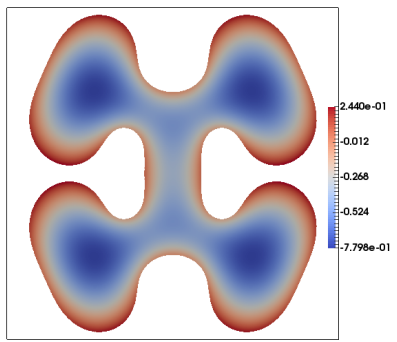

(f) $\mathrm{t}=45$

Figure 2: Simulations with $\Omega=(-5,5)^{2}, \alpha=1, \beta=0.1$ and $Q=1: \varphi_{h}$ (upper row) and $u_{h}$ (lower row).

\section{References}

[1] J. W. Barrett, J. F. Blowey, And H. Garcke, Finite element approximation of the Cahn-Hilliard equation with degenerate mobility, SIAM J. Numer. Anal., 37 (1999), pp. 286-318.

[2] J. W. Barrett, R. Nürnberg, and V. Styles, Finite element approximation of a phase field model for void electromigration, SIAM J. Numer. Anal., 42 (2005), pp. 738-772.

[3] N. Bellomo, N. Li, AND P. Maini, On the foundations of cancer modelling: selected topics, speculations, and perspectives, Math. Models Methods Appl. Sci., 18 (2008), pp. 593-646.

[4] N. Bellomo, N. K. Li, AND P. K. Maini, On the foundations of cancer modelling: selected topics, speculations, and perspectives, Math. Mod. Meth. Appl. S., 18 (2008), pp. 593-646. 


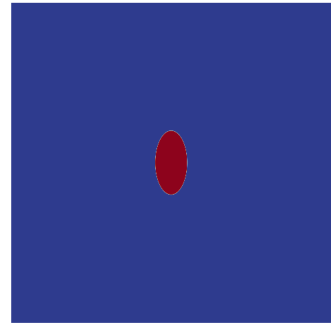

(a) $t=0$

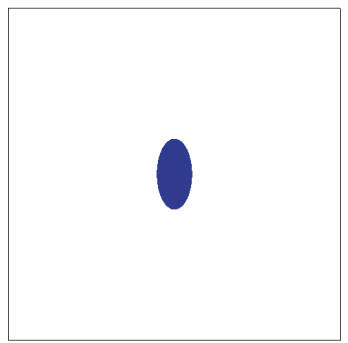

(d) $\mathrm{t}=0$

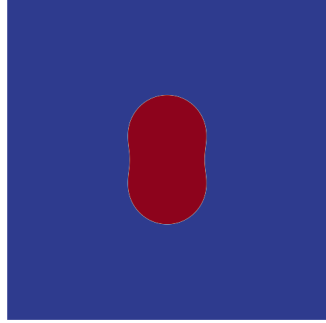

(b) $\mathrm{t}=3$

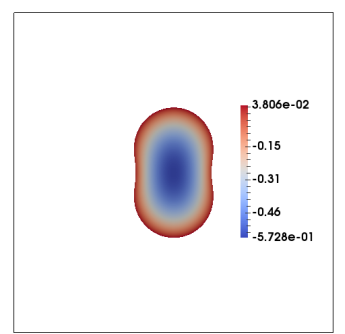

(e) $\mathrm{t}=3$

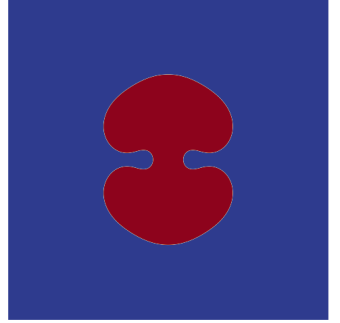

(c) $t=7$

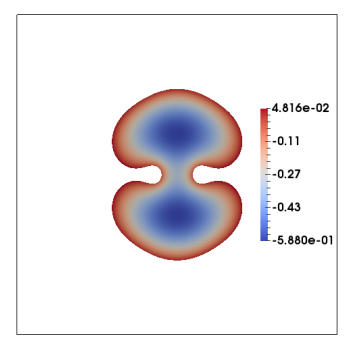

(f) $t=7$

Figure 3: Simulations with $\Omega=(-5,5)^{2}, \alpha=0.1, \beta=0.1$ and $Q=1: \varphi_{h}$ (upper row) and $u_{h}$ (lower row).

[5] J. F. Blowey And C. M. Elliott, A phase-field model with a double obstacle potential, Motion by mean curvature, ed. G. Buttazzo and A. Visintin, de Gruyter, (1994), pp. 1-22.

[6] P. Colli, G. Gilardi, And D. Hilhorst, On a Cahn-Hilliard type phase field system related to tumor growth, Discrete Contin. Dyn. Syst., 35 (2014), pp. 24232442 .

[7] V. Cristini And J. S. Lowengrub, Multiscale modeling of cancer: an integrated experimental and mathematical modeling approach, Cambridge University Press, 2010.

[8] V. Cristini, J. S. Lowengrub, And Q. Nie, Nonlinear simulation of tumor growth, J. Math. Biol., 224 (2003), pp. 191-224.

[9] K. Deckelnick And C. M. Elliott, An existence and uniqueness result for a phase-field model of diffusion-induced grain-boundary motion, Proc. R. Soc. Edinb., 131 (2001), pp. 1323-1344.

[10] K. Deckelnick, C. M. Elliott, And V. Styles, Numerical diffusion induced grain boundary motion, Interface Free Bound., 3 (2001), pp. 393-414.

[11] C. Elliott and J. Ockendon, Weak and Variational Methods for Free and Moving Boundary Problems, Pitman Publishing, 1982. 


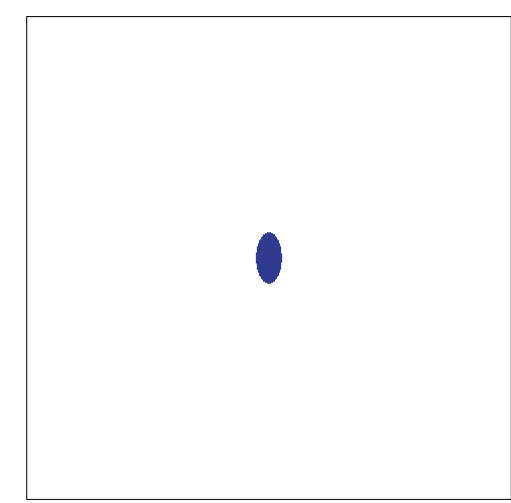

(a) $\mathrm{t}=0$

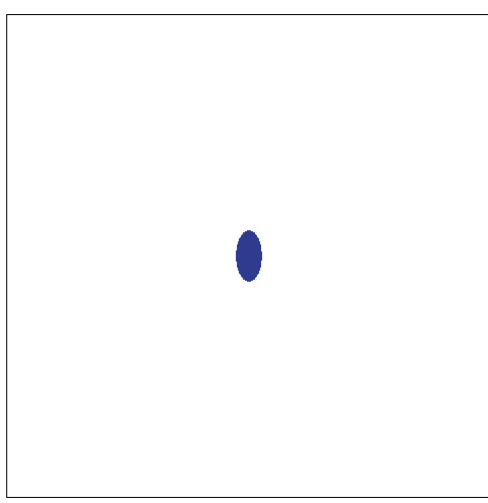

(d) $\mathrm{t}=0$

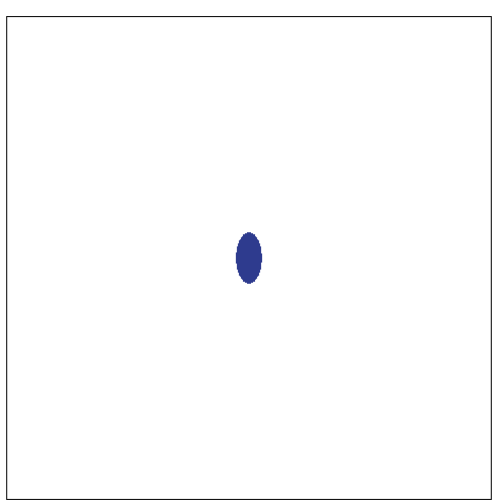

(g) $\mathrm{t}=0$

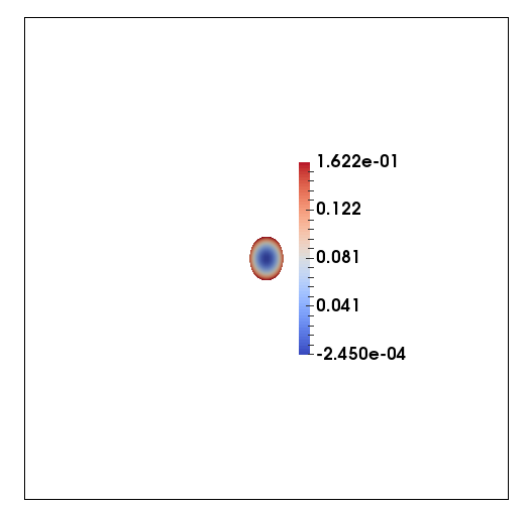

(b) $\mathrm{t}=2$

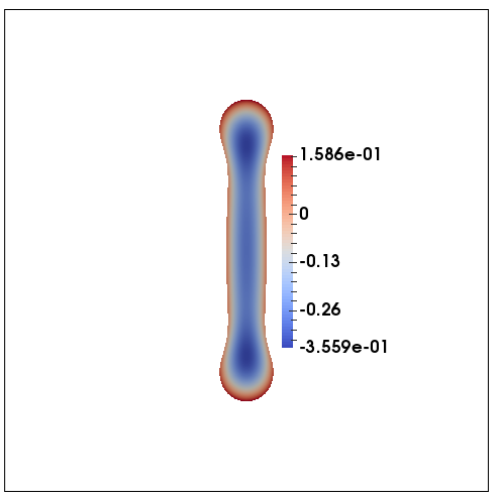

(e) $\mathrm{t}=140$



(h) $\mathrm{t}=7.5$

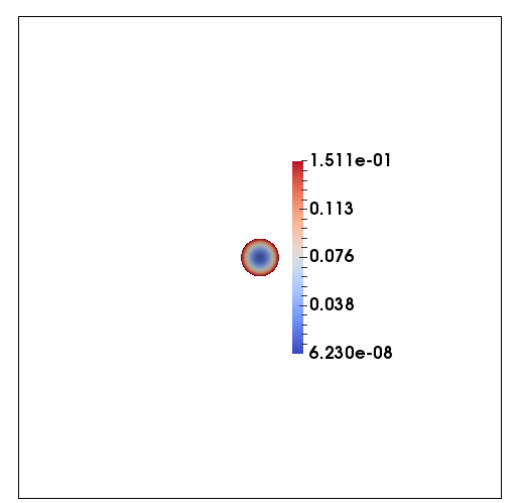

(c) $\mathrm{t}=25$

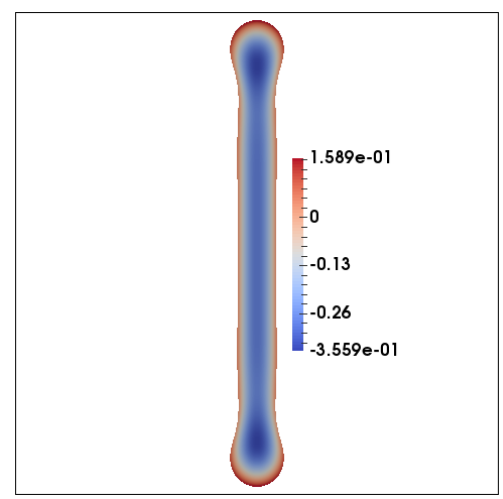

(f) $t=230$

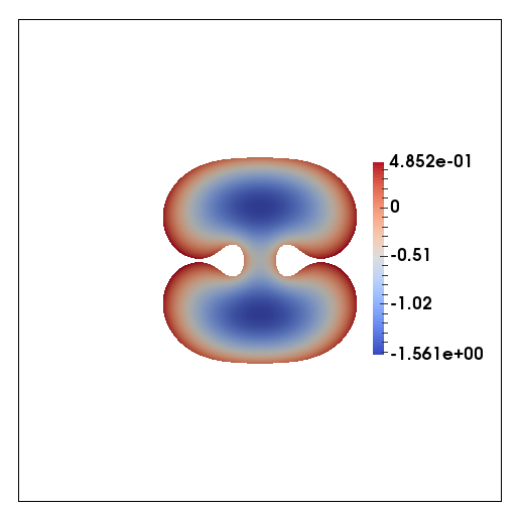

(i) $\mathrm{t}=13$

Figure 4: Simulations of $u_{h}$ with $\Omega=(-10,10)^{2}, \alpha=1, \beta=0.1$ and $Q=0.5$ (top row), $Q=0.75$ (middle row), $Q=1.5$ (bottom row). 


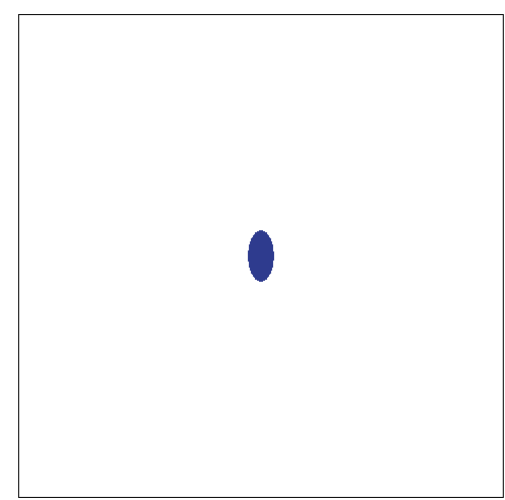

(a) $t=0$



(d) $\mathrm{t}=0$

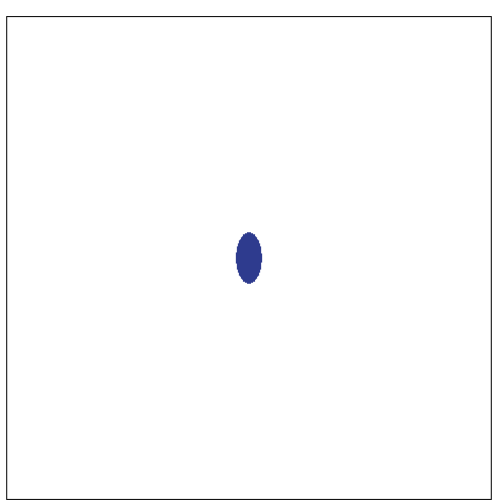

(g) $\mathrm{t}=0$

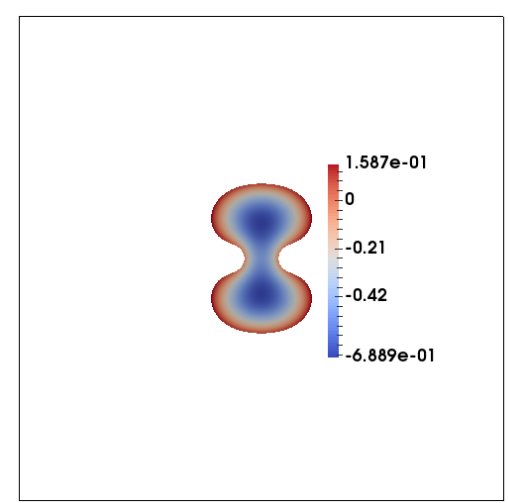

(b) $t=12.5$

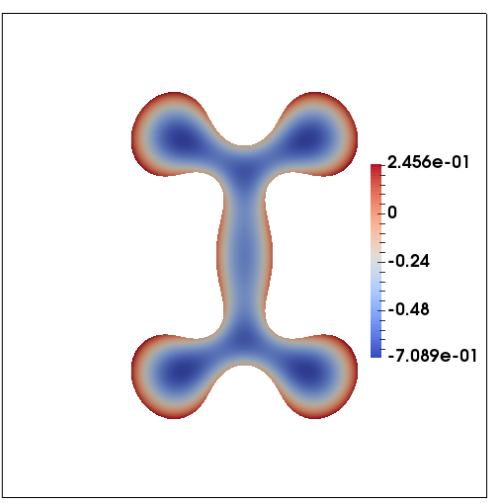

(e) $\mathrm{t}=125$

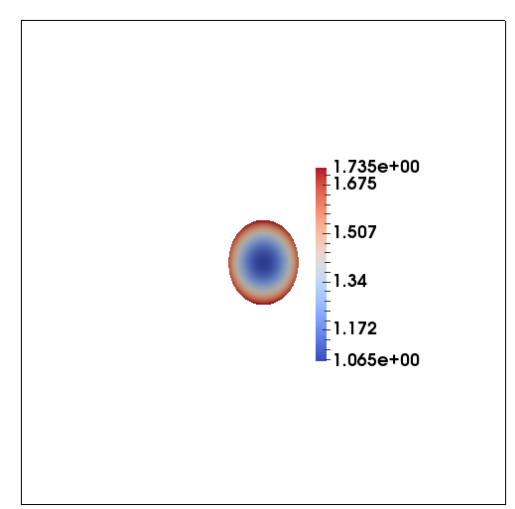

(h) $\mathrm{t}=2.5$

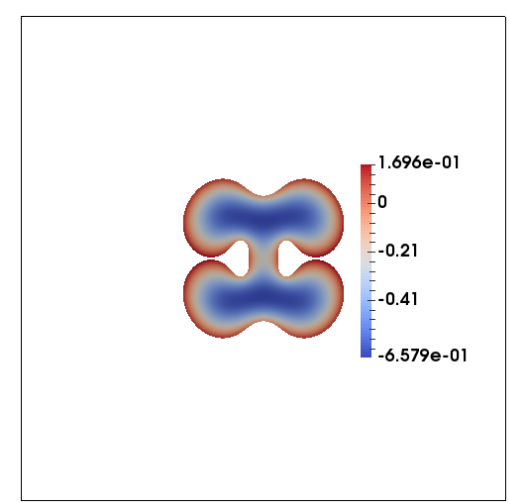

(c) $\mathrm{t}=19.5$

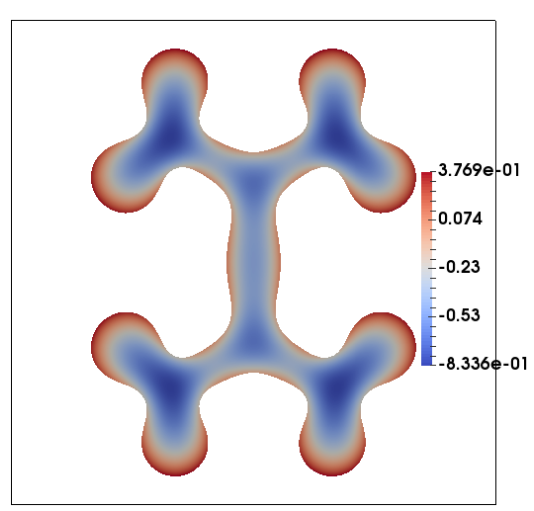

(f) $t=170$

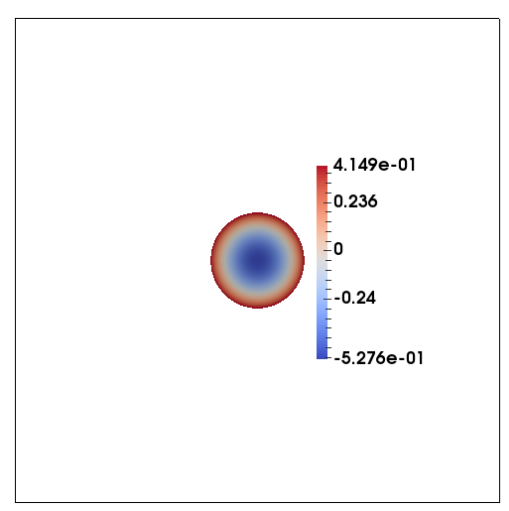

(i) $\mathrm{t}=200$

Figure 5: Simulations of $u_{h}$ with $\Omega=(-10,10)^{2}, Q=1, \beta=0.1$ and $\alpha=0.5$ (top row), $\alpha=2$ (middle row), $\alpha=7$ (bottom row). 


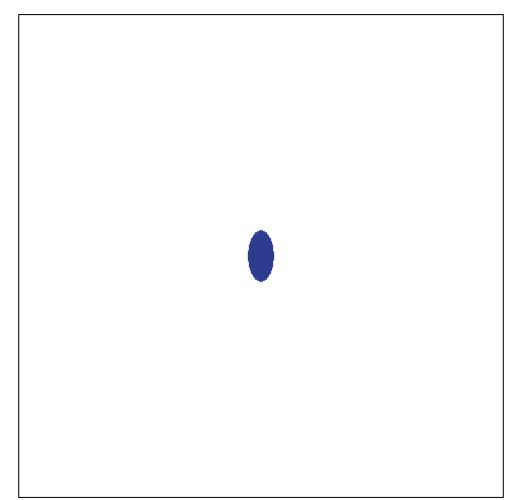

(a) $t=0$

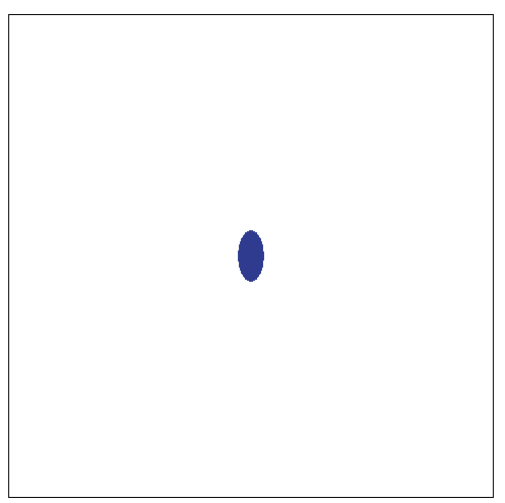

(d) $\mathrm{t}=0$

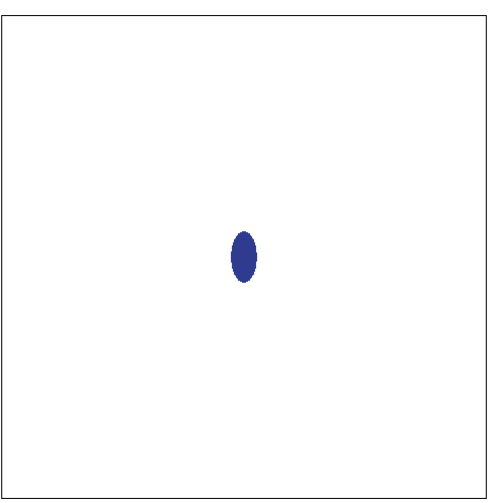

(g) $t=0$

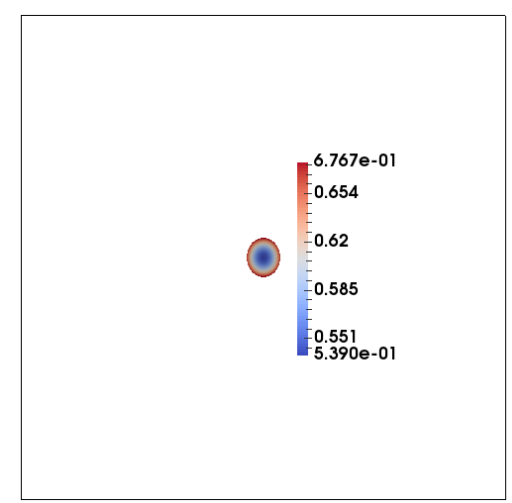

(b) $\mathrm{t}=0.5$

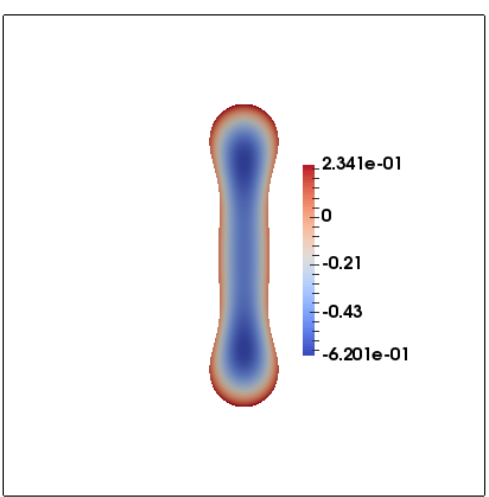

(e) $\mathrm{t}=115$

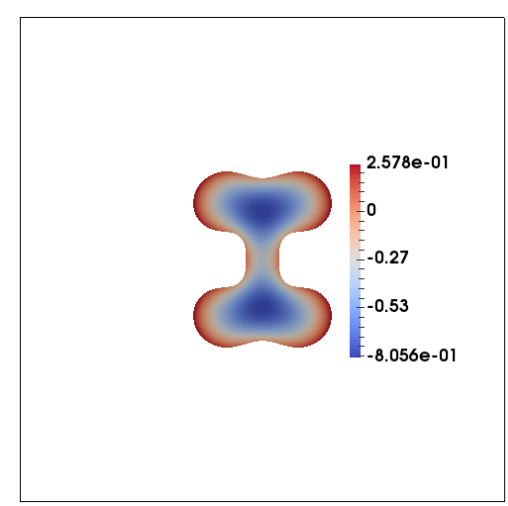

(h) $\mathrm{t}=30$

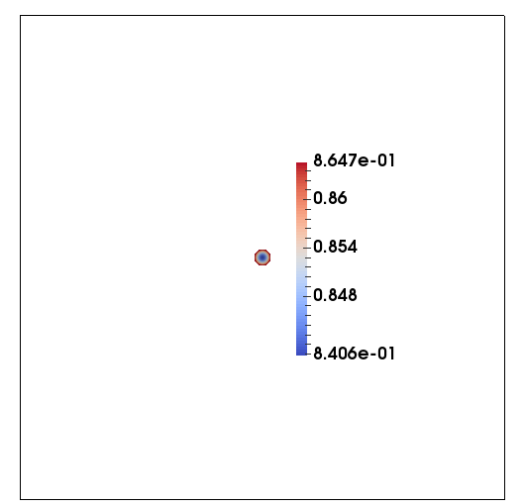

(c) $\mathrm{t}=2$

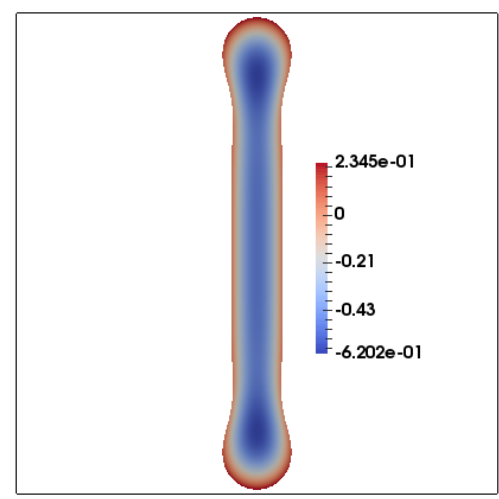

(f) $\mathrm{t}=180$

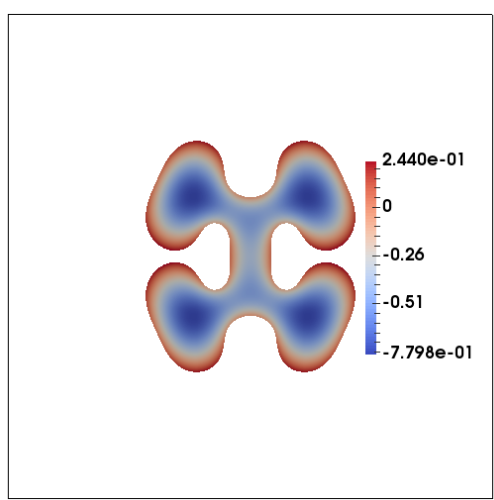

(i) $\mathrm{t}=45$

Figure 6: Simulations of $u_{h}$ with $\Omega=(-10,10)^{2}, Q=1, \alpha=1$ and $\beta=0.5$ (top row), $\beta=0.2$ (middle row), $\beta=0.1$ (bottom row). 
[12] J. Eyles, J. R. KIng, AND V. Styles, A tractable mathematical model for tissue growth, Interface Free Bound., 21 (2019), pp. 463-493.

[13] A. Friedman, Variational principles and free-boundary problems, Wiley, 1982.

[14] S. Frigeri, M. Grasselli, AND E. RoccA, On a diffuse interface model of tumor growth, Eur. J. Appl. Math., 25 (2015), pp. 215-243.

[15] H. Garcke, K. F. Lam, R. Nürnberg, And E. Sitka, A multiphase CahnHilliard-Darcy model for tumour growth with necrosis, Math. Mod. Meth. Appl. S., 28 (2018), pp. 525-577.

[16] H. Garcke, K. F. Lam, E. Sitka, And V. Styles, A Cahn-Hilliard-Darcy model for tumour growth with chemotaxis and active transport, Math. Mod. Meth. Appl. S., 26 (2016), pp. 1095-1148.

[17] H. P. Greenspan, Models for the growth of a solid tumour by diffusion, Stud. Appl. Math., 52 (1972), pp. 317-340.

[18] _ - On the growth and stability of cell cultures and solid tumours, J. Theor. Biol., 56 (1976), pp. 229-242.

[19] C. D. Hansen and C. R. Johnson, Visualization handbook, Elsevier-Butterworth Heinemann, 2005.

[20] A. Schmidt And K. G. Siebert, Design of adaptive finite element software, Springer Berlin Heidelberg, 2006. 